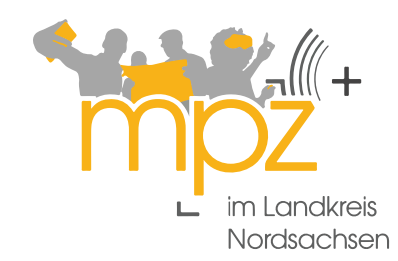

Benjamin Bigl, René Meyer und Florian Funk

Aufbruch ins Abenteuer Die virtuelle Welt der

Computerspiele

Begleitende Informationen zur gleichnamigen Ausstellung auf Schloss Hartenfels (Torgau)

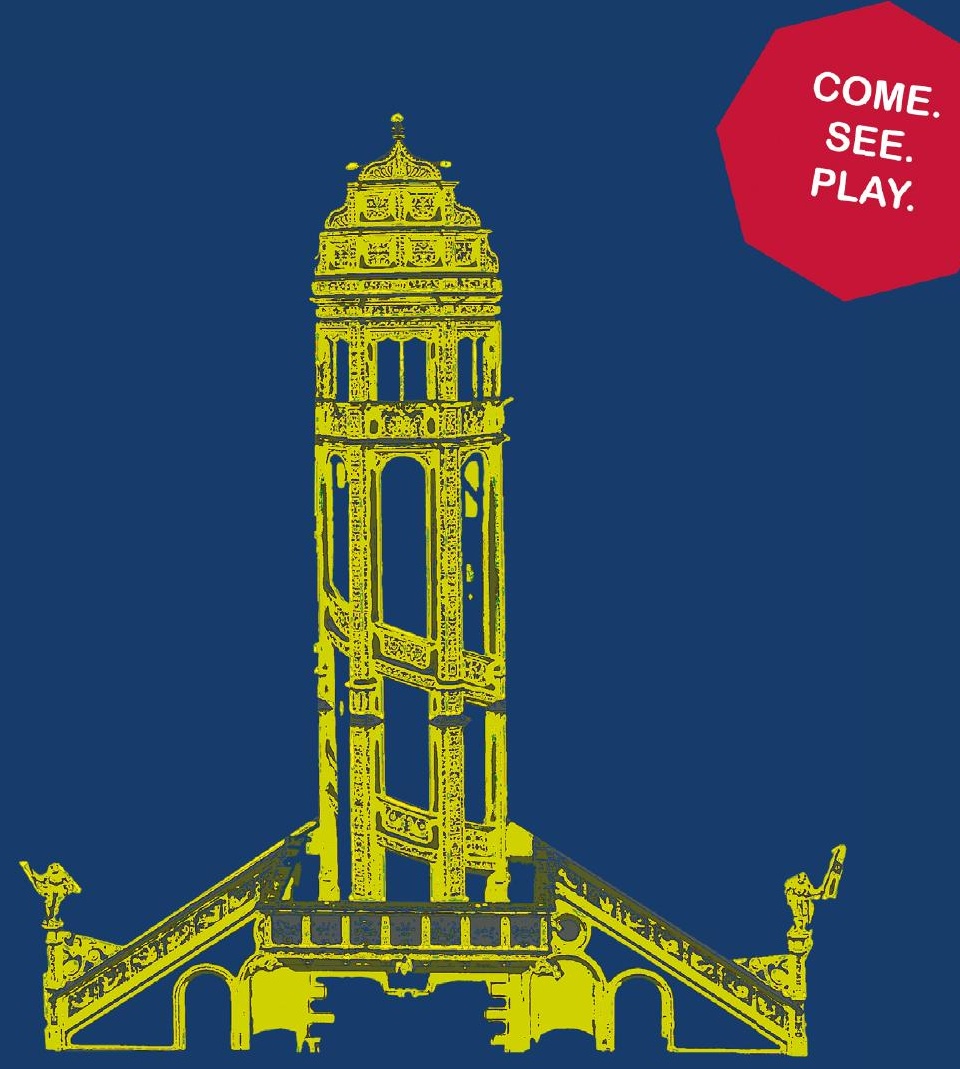




\section{Aufbruch ins Abenteuer. Die virtuelle Welt der Computerspiele.}

Begleitende Informationen zur gleichnamigen Ausstellung auf Schloss Hartenfels (Torgau).

16.12.2019-30.03.2020.

\section{Eine Kooperation von}

Medienpädagogisches Zentrump Plus

Haus der Computerspiele (Leipzig)

Kulturbetrieb Schloss Hartenfels

www.facebook.com/mpz

www.spielepower.de

www.schloss-hartenfels.de

\section{Herausgeber}

Dr. Benjamin Bigl

Medienpädagogisches Zentrumplus

Puschkinstraße 3

04860 Torgau

\section{Bildnachweis}

Landratsamt Nordsachsen, Alexander Bley; Haus der Computerspiele, René Meyer

\section{Layout}

Florian Golo Flasshoff

\section{Filmdokumentation Ausstellungseröffnung \& Informationsfilm}

Sascha Kummer, La Marée Film \& Dr. Benjamin Bigl, Medienpädagogisches Zentrum ${ }^{\text {Pus }}$

\section{Empfohlene Zitation}

Bigl, B.; Meyer, R. \& Funk, F. (2020): Aufbruch ins Abenteuer. Die virtuelle Welt der Computerspiele. Begleitende Informationen zur gleichnamigen Ausstellung auf Schloss Hartenfels (Torgau). https://doi.org/10.25366/2019.32 
Inhaltsverzeichnis

Grußwort 4

Zur Konzeption der Ausstellung 5

Aufbruch ins Abenteuer 6

Spiel-Genres 7

Alterseinstufungen der USK 8

Verantwortungsvolles Spielen 9

Die Vitrinen der Ausstellung 10

Spielkonsolen der Ausstellung 18

Kommunikation im Spiel 20

Computerspiele als virtuelle Welten 22

Faszination \& Bedeutung von Computerspielen 24

Impressionen der Ausstellungseröffnung 25

Literatur 27 


\section{Grußwort}

Liebe Besucher und Besucherinnen,

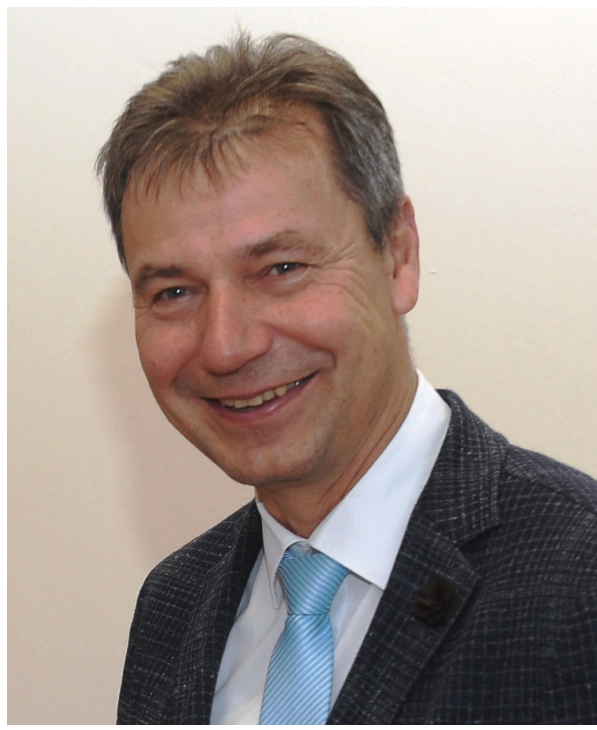

Kai Emanuel, Landrat Landkreis Nordsachsen ich freue mich sehr, Sie auf Schloss Hartenfels in Torgau zu unserer Sonderausstellung „Aufbruch ins Abenteuer. Die virtuelle Welt der Computerspiele" begrüßen zu können.

Diese Schau sucht ihresgleichen: Inmitten der traditionsreichen historischen Realität eines Schlosses können Sie eintauchen in die bunten virtuellen Welten der Computerspiele. "Aufbruch ins Abenteuer" schließt dabei nahtlos an die vorausgegangene Sonderausstellung "Mutbilder" an: Es geht auch diesmal um den zentralen Wert des sozialen und gesellschaftlichen Miteinanders.

Ungewissheit, Wettkampf und Spannung zeichnen jedes Computerspiel aus. Aber allein spielt es sich schlecht. Darum bietet Schloss Hartenfels in den kommenden Wochen für Jung und Alt die Gelegenheit, sich nicht nur miteinander zu messen, sondern auch miteinander über Spiele, die reale und die virtuelle Welt, deren Chancen, Risiken und Herausforderungen ins Gespräch zu kommen.
Die Ausstellung des Pilotprojekts MPZPlus in Kooperation mit dem Haus der Computerspiele Leipzig bietet dafür Raum. Und sie unterstütz† die Bemühungen, Schloss Hartenfels noch stärker als Bildungsort zu etablieren und über Torgaus Stadtgrenzen hinaus weiter bekannt zu machen.

Für den Landkreis sind diese Aktivitäten von besonderem Interesse. Games sind schon immer Motor und Treiber bei der technologischen Entwicklung gewesen, die Digitalwirtschaft ist ein Katalysator und attraktiv als Arbeitgeber besonders für junge Menschen. Für die anstehenden Herausforderungen der Digitalisierung können wir davon nur profitieren.

Gern lade ich Sie daher ein: Lassen Sie sich ein auf dieses Abenteuer und nehmen Sie selbst einmal den Controller in die Hand, um sich die virtuelle Welt der Computerspiele zu erschließen! Kommen Sie, schauen Sie, spielen Sie!

\section{Kai Emanuel}

Landrat des Landkreises Nordsachsen 


\section{Zur Konzeption der Ausstellung}

Etwa 524 Unternehmen in Deutschland entwickeln und vertreiben Computerspiele. Gerade für junge Menschen, die sich nach Ihrem Schulabschluss für eine Ausbildung oder den Beruf neu orientieren, ist die Games-Branche ein interessanter Arbeitgeber. In der Altersklasse der 16- bis 24-jährigen sagen dies immerhin 64 Prozent, selbst bei den 35- bis 44j-ährigen favorisieren mehr als 50 Prozent eine Tätigkeit bei Unternehmen der Digitalwirtschaft (GAME, 2020). Cloudbasiertes, flexibles und agiles Arbeiten sind in großen Unternehmen und Konzernen mittlerweile Alltag und attraktiv für Schulabgänger, die fit sind im Umgang mit digitalen Medien. Dies stellt gerade ländlich geprägte Regionen vor Herausforderungen, um einem Wegzug der Jugendlichen entgegenzuwirken, aber auch um älteren Menschen digitale Medien sowie deren Faszination näherzubringen. Mit welchem pädagogischen Konzept sollte man daher digitale Medien in einer Ausstellung präsentieren?

Hinter Computerspielen steckt zunächst gar kein bestimmtes pädagogisches Konzept. Das hat gute Gründe: Erstens sind Computerspiele interaktive Medien, die zum Zweck der Unterhaltung hergestellt und genutzt werden. Zweitens ist die Tätigkeit des Computerspielens nichts anderes als "normales" Spielen, nur eben mit Hilfe eines Computers oder einer Spielkonsole.

Der niederländische Kulturwissenschaftler Johann Huizinga versuchte in den 1930er Jahren das Spielen zu beschreiben. Er charakterisiert Spielen als eine freie Handlung, die als ,nicht so gemeint' außerhalb des gewöhnlichen Lebens stattfindet, die den Spieler völlig in Beschlag nimmt, mit der kein materielles Interesse verbunden ist, die innerhalb eines bestimmten Raumes sich vollzieht und nach bestimmten Regeln verläuft und Gemeinschaftsverbände ins Leben ruft (Huizinga, 2004). Diese Elemente treffen im Wesentlichen noch heute auf das Computerspiel zu!

Drittens machen es die Digitalisierung und Vernetzung heute auch den Nicht-Profis möglich, einen virtuellen Spielraum entstehen zu lassen, der für den Spieler Abenteuer wahr werden lässt, die vorher nur den Märchen vorbehalten waren. Diese Abenteuer sind bei jedem Spielen neu und aufregend. Diese Abenteuer muss sich der Spieler selbst und jedes Mal anders erschließen - bei diesen Abenteuern im Kopf kommt es auch nicht darauf an, ob es eine aktuelle Spielkonsole mit der neuesten Technik ist, oder doch nur eine schwarz-weiße Konsole aus den 1970er Jahren. Die Ausstellung ist daher keine Leistungsschau, sondern konzentriert sich auf ausgewählte Klassiker.

Dennoch soll nicht nur gespielt und in die Geschichte der Computerspiele eingetaucht werden. Auch der kompetente Umgang mit Computerspielen soll reflektiert werden. Dieter Baacke (1973) versteht unter dem Begriff der Medienkompetenz grundsätzlich alle Fähigkeiten, welche in einer mediengeprägten Welt zur eigenen Handlungsmacht nötig sind. Die vier Dimensionen der Medienkompetenz - Medienkunde, Mediennutzung, Mediengestaltung und Medienkritik - werden in ganz unterschiedlichen
Elementen im Rahmen der Ausstellung aufgegriffen.

Die Ausstellung unterbreitet Angebote, welche die Besucher sich selbst erschließen können und selbst erschließen sollen. Sie bietet eine Fülle an Objekten, sie gibt Auskunft zu verschiedenen Spielformen und Genres, interaktive QR-Codes bieten Kontextinformationen zu Geschichte und Kultur des Spielens, zur Spielentstehung und natürlich auch zum bewussten Umgang mit Computerspielen.

Ein rund 40-minütiger Film mit Interviews von Entwicklern, Sammlern, Designern, Forschern und Spielbegeisterten verknüpft die Themen der Exponate, erklärt wie Spiele entstehen und wirken und wie die Zukunft des Gamings wohl aussehen mag.

Als Angebot der außerschulischen Medienbildung möchte die Ausstellung auch die Älteren motivieren, sich auf unbekannte Mediensituationen einzulassen. Eltern und Pädagogen sind gefordert, nicht nur notwendige Regeln für den Umgang mit digitalen Medien zu formulieren, sondern vor allem, um mit ihren Kindern und Anderen ins Gespräch zu kommen, was und warum gespielt wird. Schauen Sie hin und spielen Sie vor allem mit. Nicht zuletzt für das gemeinsame Gespräch, den Austausch und das gemeinsame Spielen möchte die Ausstellung ein Raum der Möglichkeiten sein. Brechen Sie auf ins Abenteuer! 


\section{Aufbruch ins Abenteuer}

Computerspiele versetzen uns in zauberhafte virtuelle Welten, die sich interpretativ mit der Welt, in der wir leben, auseinandersetzen. Ob realistisch, abstrakt oder minimalistisch: Je nach Vorlieben und Fähigkeiten der Spieler erlauben Computerspiele das Eintauchen in und das aktive Erleben und Gestalten von Spielgeschichten allein, im Team oder mobil. Die Möglichkeiten sind so mannigfaltig, dass es schwerfällt, den Überblick zu behalten. Kein Wunder, schließlich sind in den vergangenen fast 50 Jahren fast 100.000 verschiedene Spiele erschienen.

\section{Faszination der virtuellen Welten}

Spiele prägen unsere Kultur und haben den Menschen von Beginn seiner Evolution an begleitet - angefangen von einfachen Würfel- oder Legespielen über Gesellschaftsspiele, Brettspiele und Sportspiele bis hin zu digitalen Computerspielen. Als interaktive Medienangebote haben Computerspiele mittlerweile alle sozialen Bereiche erreicht Mit einem Umsatz von etwa 4 Milliarden Euro pro Jahr (2018) sind sie ein immenser Wirtschaftsfaktor und attraktiv für Men-schen aller Altersgruppen. Mehr als 34 Millionen Menschen spielen in Deutschland regelmäBig, 2018 lag das durchschnittliche Alter bei 36 Jahren (GAME, 2020).

\section{Zwischen Frust und Spielspaß}

Macht, Herrschaft und Kontrolle über die eigene Handlung in der virtuellen Welt sind die drei wichtigsten Spielmotive (Fritz, 2004). Der Avatar wird so zum elektronischen Stellvertreter des Spielers im Spiel. Maßgeblich für den Spaß beim Spielen sind die Eignung des Spiels, aber auch die persönlichen Präferenzen und Kompetenzen, um den Anforderungen des Spiels gerecht zu werden. Sind Spiele zu kompliziert, ist das Spiel schnell aus. Im Idealfall können Computerspiele durch maßvolles Spielen dazu beitragen, soziale Kompetenzen zu fördern, das logische Denken in Zusammenhängen zu trainieren, sowie sich kreativ zu entfalten. Durch die Alterseinstufungen der Unterhaltungssoftware Selbstkontrolle (USK) ist für Spieler, Eltern und Pädagogen leicht erkennbar, für welches Alter das Spiel geeignet ist (USK, 2020).

\section{Die Entwicklung der Computerspiele}

Mit einem Patent von Thomas T. Goldsmith für ein "Amusement-Device" kann das Jahr 1947 als der Beginn der Computerspielentwicklung angesehen werden. Das Gerät erforderte sehr viel mehr Fantasie als heute, es vermochte lediglich bewegbare, grün leuchtende Punkte darzustellen. Die Vorläu- fer heutiger Computerspiele sind die Automatenspiele der 1970er und 1980er Jahren, die in Hotels und Restaurants äußerst beliebt waren. Während der klassische PC ursprünglich nur für Büroaufgaben konzipiert war, fand 1972 mit der ersten Spielkonsole (Magna-vox) ein Wandel der Spielkultur statt - gespielt wird seitdem nicht mehr ausschließlich am Schreibtisch, sondern im Wohnzimmer vor dem Fernseher mit anderen. Im mobilen Alltag sind Computerspiele seit dem Game Boy von Nintendo (1989) allgegenwärtig, Smartphones haben die transportablen Spielkonsolen jedoch fast verdrängt. Egal ob im Bus, auf Plätzen, in Parks oder Cafés: auch vernetztes Spielen online ist ein Abenteuer, was jederzeit auch mit anderen möglich ist (Bigl, 2016; Forster, 2009). 


\section{Spiel-Genres}

Casual Games sind besonders auf Spielkonsolen populäre Spiele und umfassen Brett-, Karten-, Rhythmus-und Partyspiele wie "Sing Star". Arcade-Spiele wie "Pong!" sind einfache, rundenbasierte Geschicklichkeitsspiele und entstammen der Zeit der Spielautomaten. In Jump'n'Run-Spielen wie bspw. „Super Mario" muss die Spielfigur auf verschiedenen Ebenen Gegenstände einsammeln, die Punkte bringen. In Adventures steht das Erleben einer Geschichte im Mittelpunkt, oft wird die Spielfigur nur durch Mausklicks gesteuert - "Monkey Island" ist ein Klassiker dieser Point-and-Click-Adventures. Die Shooter für Erwachsene werden als rasante Action-Spiele meist in der Ich-Perspektive gespielt, was besonders ins Spiel hineinzieht. Die Story ist dabei häufig nebensächlich, wichtiger ist die detailreiche Darstellung des Geschehens sowie des wahrgenommenen dreidimensionalen Raumes, in dem sich der Spieler mit Maus und Tastatur bewegt (oftmals WASD), In Rollenspielen steht die Entwicklung des Charakters der eigenen Spielfigur (Avatar) im Mittelpunkt. Dies geschieht über das Lösen von Aufgaben (Quests) in einer Fantasiewelt. Eine Sonderform sind MMORPGs (Massively Multiplayer Online Role Playing Games) wie "World of Warcraft", wo tausende Spieler sich in so genannten Gilden (Gruppen) verbünden.
In Strategie- und Simulationsspielen steht aus der Vogelperspektive der Aufbau bspw. einer Zivilisation im Mittelpunkt (bspw. im Spiel "Civilization"). Sport- und Rennspiele wie "Need for Speed" erfordern Konzentration, Geschicklichkeit und Schnelligkeit. Besonders im Bereich der E-Sport-Spiele (bspw. "League of Legends", "FIFA International Soccer") etablieren sich zunehmend eigene Verbände und Ligen mit öffentlichen Wettkämpfen (bspw. Dreamhack in Leipzig).

\section{Datenschutz \& kostenpflichtige Spielinhalte}

Vorsicht ist geboten bei angeblich kostenlosen Spielen und Apps, bei denen innerhalb des Spieles kostenpflichtige Zusatzangebote wie bspw. Ausrüstungsgegenstände erworben werden können oder die Glücksspiel-Elemente enthalten (bspw. "Coin Master"). Insbesondere bei Spiele-Apps kann für einen kompetenten Umgang mit diesen Spielen auch das Thema Datenschutz relevant werden, wenn das Spiel Berechtigungen auf den Zugriff zu persönlichen Daten des Smartphone-Benutzers einfordert (u.a. auf das Adressbuch oder den Standort). Hier sind insbesondere Eltern, Angehörige und Pädagogen gefordert, Kinder und Jugendliche für diese Themen fit zu machen.

\section{Alterseinstufungen}

Für die Alterseinstufung von Computerspielen ist in Deutschland die Unterhaltungssoftware Selbstkontrolle (USK) zuständig. Jedes Spiel, welches in Deutschland verkauft wird, unterliegt dieser Prüfung und Einstufung. Dafür gibt es festgelegte Kriterien, die in Zusammenarbeit mit den Obersten Landesjugendbehörden und unter Berücksichtigung der Bundesprüfstelle für jugendgefährdende Medien (BPJM) entwickelt wurden und erklären, welche Aspekte sich negativ auf die Entwicklung von Kindern und Jugendlichen auswirken könnten. Die Alterseinstufungen der USK finden sich auf jeder Spieleverpackung (§ 14 JuSchG).

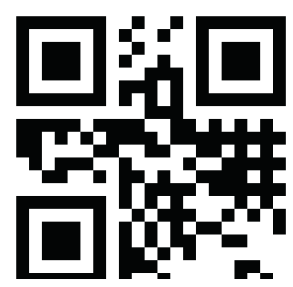

www.usk.de 


\section{Alterseinstufungen der USK}
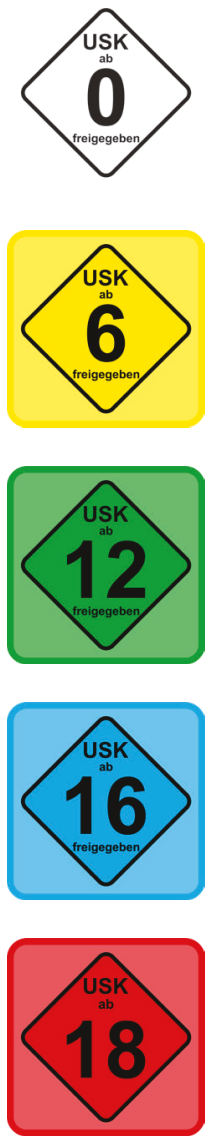

\section{ohne Altersbeschränkung}

familienfreundliche Spiele (bspw. Geschicklichkeits- und Gesellschaftsspiele, Sportspiele, Jump 'n` Runs)

\section{ab 6 Jahren}

überwiegend familienfreundliche Spiele, die bereits spannender und wettkampfbetonter ausfallen dürfen (bspw. Rennspiele)

\section{ab 12 Jahren}

schon deutlich kampfbetontere Spiele, die Spielszenarien werden oft von einer Handlung gerahmt und bieten Distanzierungsmöglichkeiten (bspw. Adventure, Strategie- und Rollenspiele)

\section{ab 16 Jahren}

auch Gewalthandlungen werden gezeigt, häufig bewaffnete Kämpfe mit einer Rahmenhandlung (bspw. militärische Strategiespiele)

\section{ab 18 Jahren}

auf Grund der gewalthaltigen Spielkonzepte und ihrer bedrohlichen Atmosphäre sind diese Spiele ausschließlich für Erwachsene freigegeben (bspw. Ego-Shooter, Action-Adventures) 


\section{Verantwortungsvolles Spielen}

Markige Überschriften wie "Je brutaler die Spiele, desto schlechter die Schulnoten" (Main Post, 15.10.2013) zieren immer dann die Titelseiten von Tageszeitungen, wenn Computerspiele in der Kritik stehen, sich negativ vor allem auf die Entwicklung von Kindern und Jugendlichen auszuwirken. Die Frage, wie Computerspiele wirken, ist jedoch äußerst komplex und nicht einfach zu beantworten. Viele Faktoren wie bspw. die Persönlichkeit des Spielers, seine individuellen Vorerfahrungen, seine allgemeine $\mathrm{Me}$ dienkompetenz, aber auch soziale Faktoren sowie natürlich die Art und die Beschaffenheit des Spieles bestimmen letztlich das Erleben von virtuellen Computerspielwelten.

Verschiedene Anlaufstellen von Organisationen, Einrichtungen und Vereinen bieten daher konkrete Unterstützung und medienpädagogische Orientierung im Spiele-Dschungel und helfen bei der Spielauswahl.
In Sachsen unterstütz† die Koordinierungsstelle Medienbildung (KSM) die Netzwerkbildung und Unterstützung in der Medienbildungslandschaft in Sachsen. Die KSM macht die medienpädagogischen Angebote in Sachsen sichtbar und vernetzt außerschulische Akteurinnen und Akteure, berät die sächsische Bevölkerung und weist auf wichtige Informationen im Bereich Medienbildung hin.

Empfehlenswerte Fachbücher (unter Literatur) geben einen fundierten wissenschaftlichen Überblick zum Stand der Forschung zu Nutzung und Wirkung von Computerspielen und (digitalen) Medien.

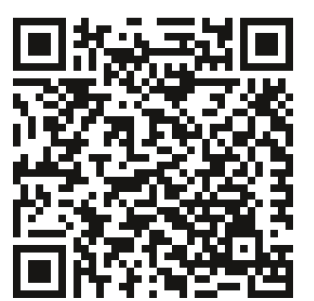

www.medienbildung.sachsen.de

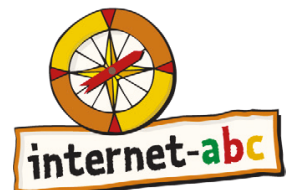

www.internet-abc.de

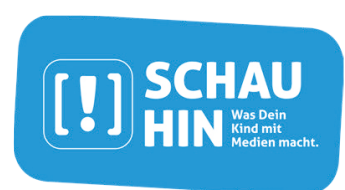

www.schau-hin.info

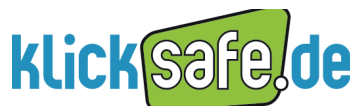

www.klicksafe.de

\section{spielbar.de}

www.spielbar.de 


\section{Die Vitrinen der Ausstellung}

\section{LCD-Spiele und Miniatur-Automatenspiele}

Neben Quarzuhren, Taschenrechnern und tragbaren Kassettenspielern sind in den achtziger Jahren tragbare LCD-Spiele ein beliebter Zeitvertreib. Der Game Boy ist noch nicht erfunden (er erscheint erst 1990 in Europa) und so vergnügt man sich unterwegs mit kleinen Geräten, die ein einfaches Spie eingebaut haben. Der Hintergrund ist durch farbige Folien gestaltet. So fällt es weniger auf, dass sich das eigentliche Spielgeschehen auf einige Elemente beschränkt, die sich auf festgelegten Positionen bewegen.

Manche Geräte haben zwei oder drei Bildschirme, die verschiedene Levels darstellen. Die Geräte sind nicht so teuer, 20 bis 50 DM und finden ihren Weg auch in die DDR.

Besonders gefragt ist die Game\&Watch-Serie von Nintendo. Sie hat, der Name deutet es an, neben dem Spiel eine Uhr mit Wecker integriert. Sie ist in Westdeutschland auch unter dem Handelsnamen tricOtronic bekannt. Die Serie wird in der Sowjetunion nachgebaut und unter der Marke Elektronika im Handel angeboten. Auch diese Spielzeuge werden gern mit in die DDR gebracht; zu Preisen um die 20-40 Rubel - ungefähr 3060 Euro nach heutiger Rechnung.

$\mathrm{Ab}$ den neunziger Jahren sind LCD-Spiele mit inrer einfachen und starren Unterhaltung nicht mehr zeitgemäß. Die Ära der Handhelds mit Wechselmodulen wie der Game Boy hat begonnen. Dennoch werden noch heute LCD-Spiele in großer Zahl produziert. Sie sind für wenige Euro zu haben und wer- den gern als Beigabe mitgegeben, etwa zu einer Kinderzeitschrift.

Die großen Brüder der LCD-Spiele sind Table Tops. Die Form der Miniatur-Automaten erinnert an die Geräte in Spielhallen. Sie haben nur ein Spiel eingebaut. Heute hat die in Vergessenheit geratene Form Konjunktur durch eine neue Generation von Mini-Arcades, die jeweils zahlreiche Spiele emulieren.

Eines der originellsten Geräte ist sicher der Virtual Boy von Nintendo, der bereits 1995

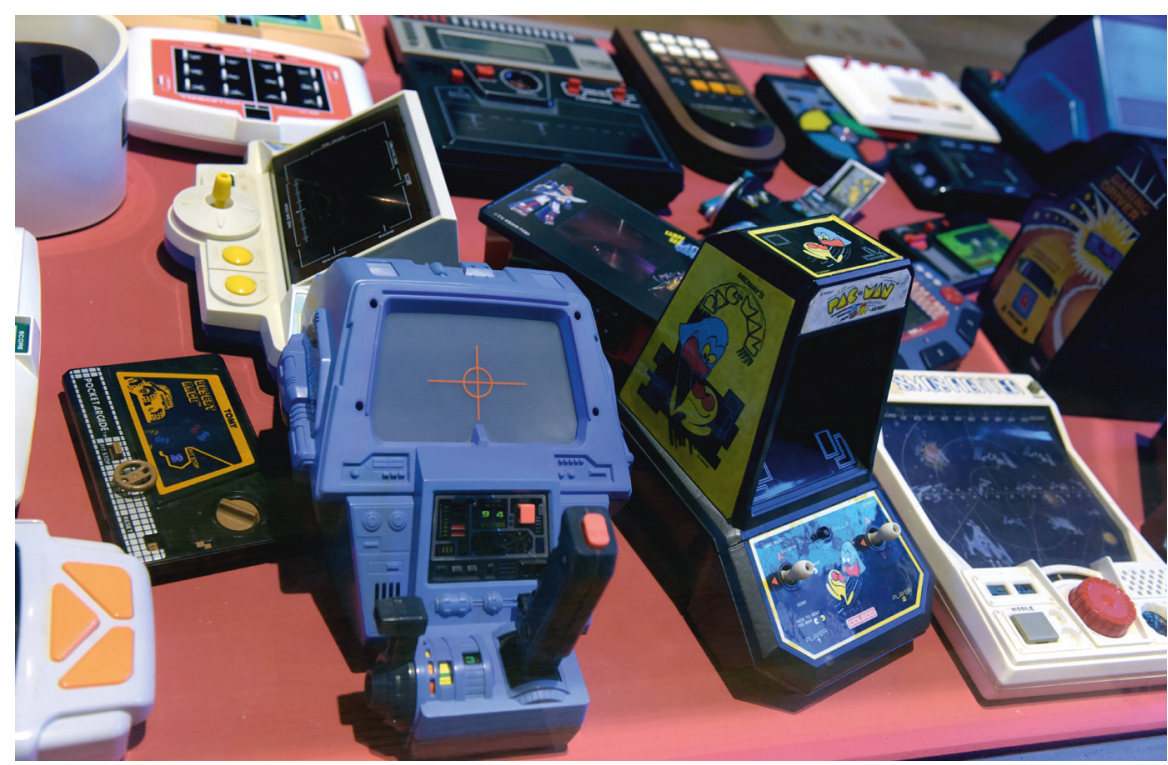

ein 3D-Bild liefert, allerdings nur zweifarbig rot und schwarz. Der Virtual Boy ist ein Misserfolg und erscheint nur in Japan und den USA, aber nicht in Europa.

Ähnlich ungewöhnlich ist die im gleichen Jahr erschienene Headset-Variante der R-Zone von Tiger Electronics. Sie projiziert das Bild über eine transparente Linse ins Auge des Spielers. 


\section{Jahre PlayStation}

Ursprünglich wollten Sony und Nintendo gemeinsam eine Konsole entwickeln. Als sich Nintendo zurückzieht, beschließt Sony, allein weiterzumachen. Die Voraussetzungen sind gegeben: Sony hat einerseits viel Erfahrung mit Hardware, entwickelt etwa schon in den 1980ziger Jahren einen Heimcomputer (namens HitBit), und ist andererseits neben Musik auch als Verleger von Spielen aktiv.

Basis der Playstation ist die damals neue CD-Technik. Sie bietet weitaus mehr Speicher für Sprachausgabe und Videos als die bisherigen Steckmodule. Die leistungsfähigen Chips erlauben eine 3D-Graphik, wie man sie bisher nur vom PC her kennt. Sony übernimmt eine Reihe von Entwicklern wie Psygnosis. Dadurch entstand eine große Anzahl von Spielen. Ein Grund für den Erfolg ist das Zuwenden an neue Käuferschichten. Die PlayStation ist "cooler" als die bunte Nintendo-Welt und zieht verstärkt Erwachsene an.

Die PlayStation wird zur zweiterfolgreichsten TV-Konsole überhaupt. Nur ihr eigener Nachfolger, die PlayStation 2, verkauft sich mehr. In einer Vitrine ist eine originale Playstation im Airbrush-Design zu sehen, von der nur wenige Exemplare gestaltet wurden, eine
PlayStation 4 als graue Jubiläumsausgabe, die 2014 zum 20. Geburtstag erschien, eine PSOne als abgespeckte Variante der PlayStation mit aufgestecktem Bildschirm, eine PocketStation (eine nur in Japan erschienene Mischung aus Memory Card und LCDSpiel als Zubehör) und die neue PlayStation Classic, die 20 Spiele fest eingebaut hat.

Unter den Exponaten: Eines der damals sehr beliebten MogelPower-Bücher mit Schummeltricks.

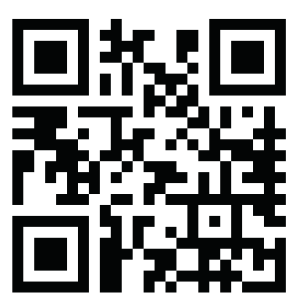

www.mogelpower.de

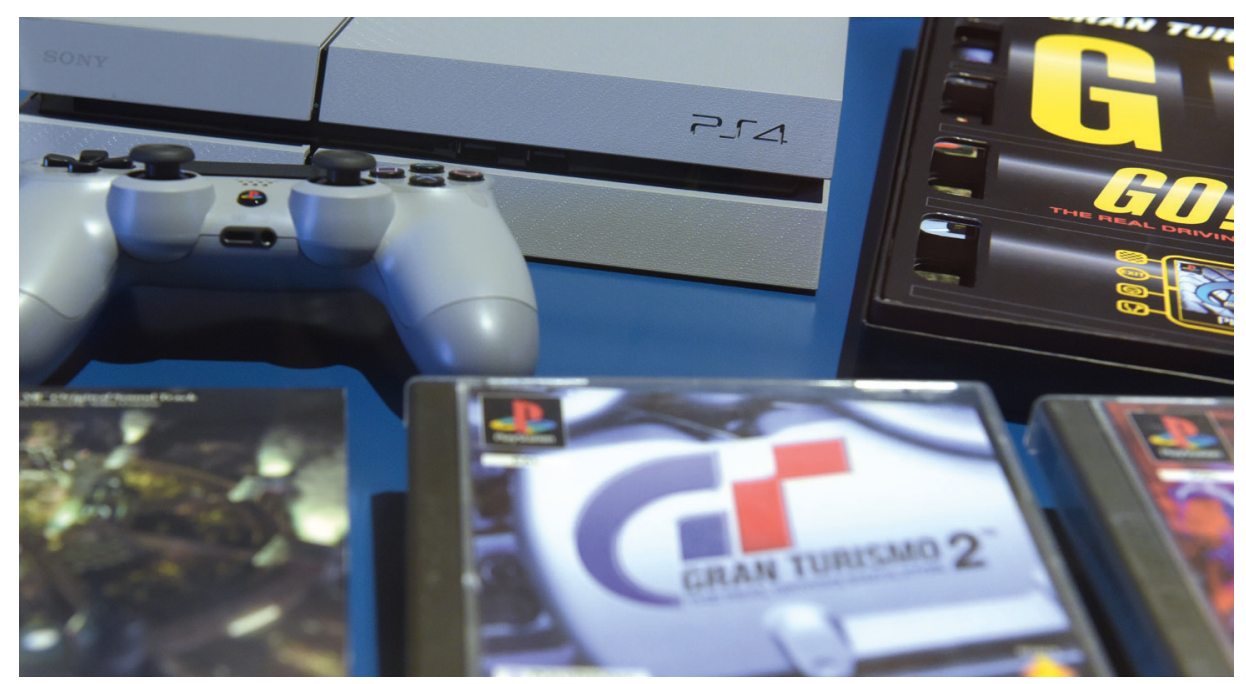




\section{Jahre Game Boy}

Am 21. April 1989 erscheint der Game Boy in Japan. Nach Deutschland kommt er erst im September 1990. Mit 120 Millionen verkauften Geräten ist er das dritterfolgreichste Spielsystem, hinter dem Nintendo DS und der PlayStation 2. Zwar bieten Mitbewerber wie Atari Lynx und SEGA Game Gear bereits Farbe, aber der Game Boy ist handlicher, hat deutlich längere Batterie-Laufzeiten und mehr und bessere Spiele. Allen voran natürlich "Tetris” und die Mario- und Zelda-Teile. Später kommt „Pokémon” dazu.

\section{Versionen}

1989 Game Boy (in Deutschland 1990)

1996 Game Boy Pocket mit kleineren Maßen

1996 Pokémon - rote und grüne Edition (in Deutschland 1999)

1997 Game Boy Light mit Hintergrundlicht (nur in Japan erschienen)

1998 Game Boy Camera und Game Boy Printer

1998 Game Boy Color mit Farbe

2001 Game Boy Advance mit neuer Technik und kleineren Modulen

2003 Game Boy Advance SP mit Klappe und Beleuchtung

2005 Game Boy Micro

2004 Nintendo DS mit zwei Bildschirmen (in Deutschland 2005)

2011 Nintendo 3DS mit 3D-Effekt

2012 Nintendo 3DS XL mit größeren Maßen

2013 Nintendo 2DS ohne Klappe und ohne 3D

2014 New Nintendo 3DS / 3DS XL mit mehr Tasten und mehr Leistung

2017 New Nintendo 2DS XL

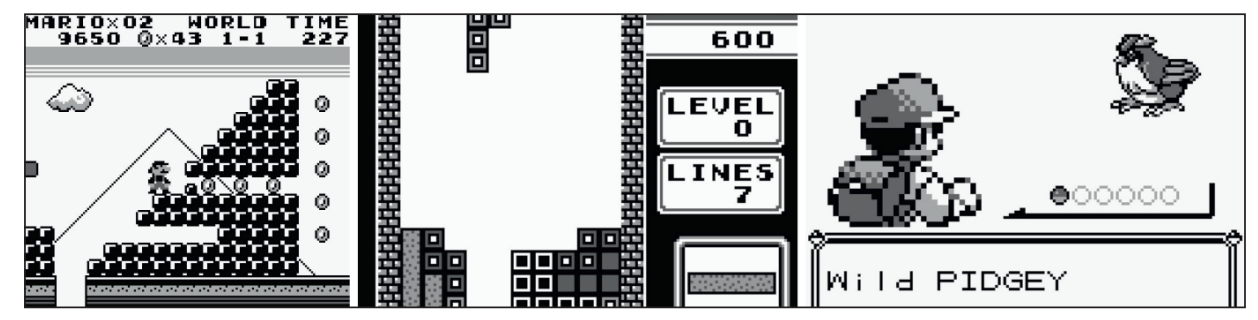

Screenshots Game Boy 


\section{Jahre Moorhuhn}

Die erfolgreichste deutsche Spielserie? "Die Siedler"? „Anno"? Weit gefehlt: das "Moorhuhn" wurde nicht nur millionenfach aus dem Netz geladen, sondern erschien auch in rund vierzig Ausführungen im Handel. Zunächst als Ballerspiel; später auch in Adventures, Rennspielen, Jump'n'Runs, in Sportarten wie Fußball, auf dem Mobilphone. Und im Ausland unter dem Namen Crazy Chicken.

Die Geschichte begann 1997, als Ingo Buchholz von der Hamburger Werbeagentur $V$ und $B$ für seinen Kunden Johnnie Walker eine neue Kampagne überlegte. Jedes Quartal hatte eine neue Aktion und für den Herbst kam inm der Gedanke einer visuellen Moorhuhn-Jagd in den schottischen Highlands. Der ursprüngliche Gedanke war eine Apparatur, doch er wurde als zu aufwendig empfunden. Promoter sollten mit Notebooks abends durch Kneipen ziehen und die Gäste mit einem kleinen Spielchen locken.

Für die Umsetzung wurde Frank Ziemlinski und sein Spielestudio Art Department ins Boot geholt, das damals viele Werbespiele entwickelte und auch schon für $\mathrm{V}$ und $\mathrm{B}$ ein kleines Spielchen für eine andere Alkohol-Marke umgesetzt hatte. Die eigentliche Programmierung erfolgte durch das niederländische Studio WITAN - streng genommen wurde das bekannteste deutsche Spiel gar nicht in Deutschland entwickelt. Von Ingo Mesche stammt die berühmte Moorhuhn-Figur. Er hatte sie schon Jahre zuvor für ein nie erschienenes Amiga-Spiel entworfen.
Im Herbst 1998 ging es auf Kneipen-Tour. Doch WITAN hatte eine fast fertige Version bereits im August bei der Demo-Party Bizarre unter dem Namen Kippen Schieten vorgestellt, wo es gar mit einem Preis ausgezeichnet wurde. Obwohl man das Spiel kostenlos aus dem Netz laden konnte, blieb es zunächst ein Ladenhüter. Erst 1999 wurde aus dem simplen Ballerspiel ein Erfolgsschlager.

Ein Berliner Radiosender nahm es in sein Programm auf, es war in der Harald-SchmidtShow zu sehen und im Frühjahr 2000 landete es gar auf der Titelseite der BILD: „Volkskrankheit Moorhunn".
Irgendwie kam alles zusammen. Viele Haushalte hatten bereits einen Internet-Anschluss, das Spiel war kostenlos, klein genug auch für einen E-Mail-Anhang, leicht zu verstehen und es hatte eine Bestenliste. Es gab Strategien für viele Punkte, und das Glück spielte eine gewisse Rolle so dass man sich immer wieder daran versuchen konnte.

Der Nachfolger von Art Department, Phenomedia, erkannte die Chance und zog die Marke groß auf. Und irgendwie geschah es, dass das Interesse an der Marke nie abriss. Der jüngste Sproß: Moorhuhn VR.

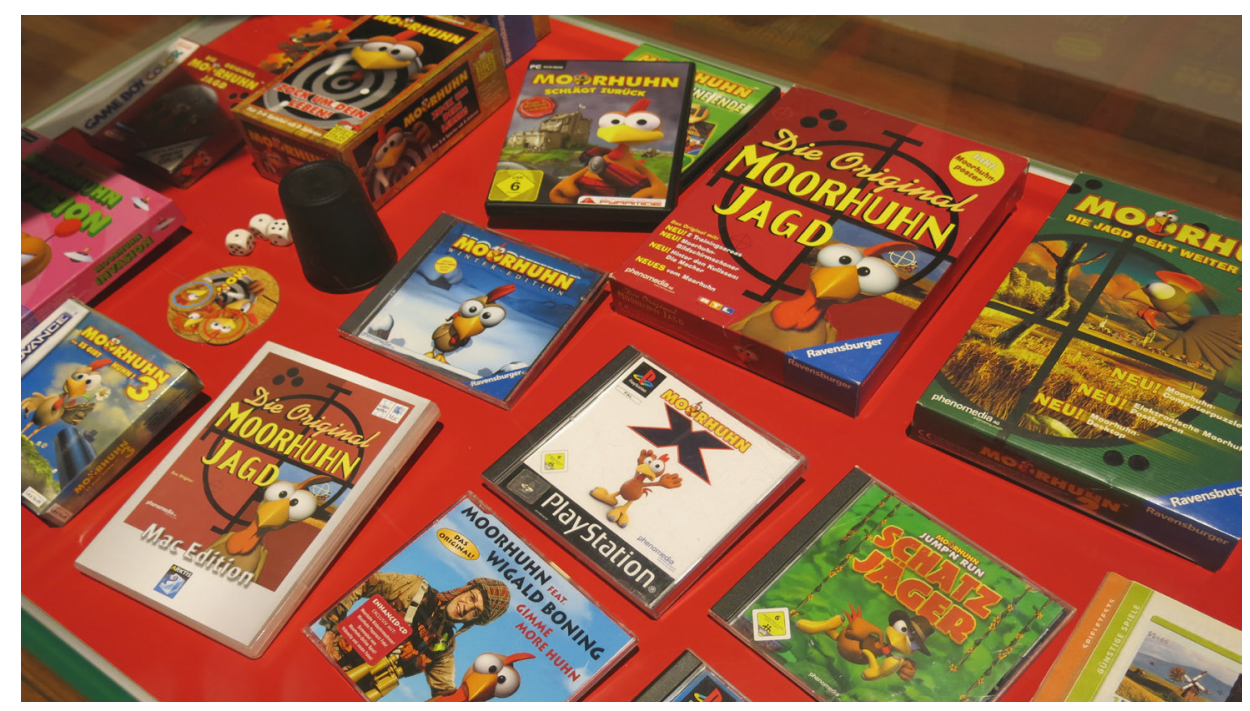




\section{Commodore CBM 3032}

1975 erscheinen die ersten bezahlbaren Heimcomputer wie der Altair 8800 und der IMSAI 8080. In der Grundausstattung kann man nicht viel damit anstellen; die Geräte bestehen aus LEDs als Anzeige und aus Kickschaltern für mühsame Programmierung im Maschinen-Code. Sie werden häufig als Bausatz verkauft und müssen erst zusammengesetzt werden. Doch beide Computer sind kompatibel zueinander und erweiterbar. Das ist die Geburtsstunde des ersten Heimcomputer-Standards, für den zahlreiche, oftmals frisch gegründete Unternehmen Zubehör anbieten.

Ebenfalls 1975 wird Microsoft gegründet. Das erste Produkt ist die Umsetzung der Programmiersprache BASIC für den Altair. Sie macht nicht nur den störrischen Bastelcomputer für Einsteiger zugänglich. BASIC wird die übergreifende Sprache für alle Heimcomputer.

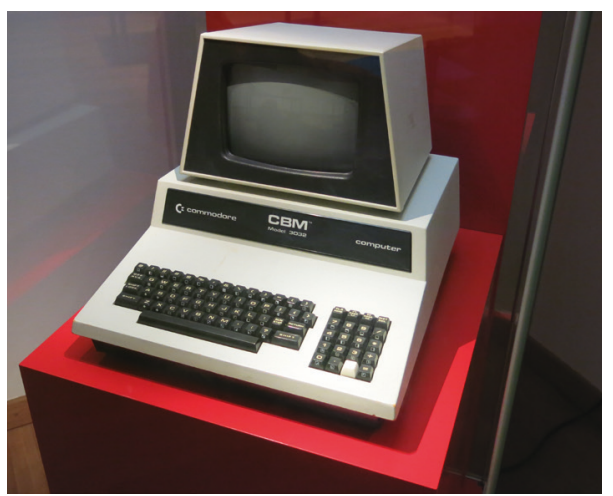

Ab 1977 gibt es betriebsbereite Heimcomputer mit Gehäuse und Tastatur zu kaufen. Zunächst den Apple II, den TRS-80 von Radio Shack und den Commodore PET, der einen Bildschirm gleich eingebaut hat. Aus dem PET erwächst eine ganze Serie von Rechner-Modellen, die Commodore mit der Einführung in Europa in CBM umbenennen muss - den Namen PET hat sich schon Philips gesichert. Die Reihe ist sehr erfolgreich und wird vor allem in Betrieben und Schulen eingesetzt.

Der in der Ausstellung gezeigte CBM 3032 ist 40 Jahre alt - von 1979. Er hat einen Arbeitsspeicher von 32 Kilobyte, was ungefähr der Zeichenmenge von zehn A4-Seiten entspricht. Zum Speichern und Laden wird ein Kassettenrekorder benötigt. Er speichert Programme als Töne auf herkömmlichen Tonbandkassetten. Auch teure Diskettenlaufwerke gibt es, Festplatten sind für Büros und Haushalte noch unerschwinglich.

Durch den Grünmonitor und ohne einzeln ansteuerbare Bildpunkte entstehen nur einfache Spiele, die aus Zeichen bestehen: Textadventures ohne Graphiken, nur mit beschreibenden Texten und Befehlen wie GEHE NORD, ÖFFNE TÜR oder Wirtschaftsspiele, bei denen Waren gekauft und verkauft werden. Und Geschicklichkeitsspiele, bei denen die Levels und die Spielfiguren durch Buchstaben und Zeichen wie \% dargestellt werden. Ein sogenannter Parser verarbeitet diese Eingaben.

\section{Adventures}

Das Adventure ist eines der ältesten Spielegenres. Seinen Namen erbt es vom ersten Vertreter, "Collosal Cave Adventure", das bereits Anfang der siebziger Jahre entsteht und nur Text enthält, keine Bilder. Das Programm beschreibt eine Umgebung und der Spieler muss seine Anweisungen eintippen, um das Abenteuer voranzutreiben. Das ist jetzt fünf Jahrzehnte her, die Zeit, als Großrechner regieren, aber die Prinzipien, die das Spiel charakterisierten, gelten heute noch

Ein Adventure ist den strengen Regeln unterworfen, die vom Programmierer festgelegt sind. Die Welt, in die der Spieler eingeladen wird, mag flexibel erscheinen, ist aber in Wirklichkeit relativ starr. Probleme lassen sich meist nicht so lösen, wie die Phantasie vorschlägt, sondern nur so, wie es vorgesehen wurde. Die einzige Wahlmöglichkeit besteht meist darin, Teilaufgaben in verschiedener Reihenfolge anzugehen. Das frustriert manchmal, regt aber auf der anderen Seite die grauen Zellen an. Das Knobeln, wie es nun weitergeht, welcher Hinweis verfolgt wird, welcher Gegenstand wie verwendet wird, das macht Adventures beliebt. Die Firma Infocom greift die Idee von "Adventure" für ihre Trilogie „Zork" auf und vermarktet sie für Heimcomputer. Dank guter Geschichten und eines leistungsfähigen Parsers wird Infocom zum Marktführer. Vor allem: Man richtet es so ein, dass jedes neue Infocom-Spiel auf einer Vielzahl von Computertypen läuft. Bis heute. 
Die nächste Entwicklungsstufe isł die Anreicherung um Grafiken. Zwar müssen immer noch Befehle eingetippt werden, aber die Beschreibungen werden um Bilder ergänzt. Vorreiter ist das junge Studio Sierra im Jahre 1980, das mit Reihen wie „King's Quest” groß wird.

1987 übernimmt mit Lucasfilm Games (später LucasArts) ein anderes Unternehmen die Führungsrolle für Innovationen: „Maniac Mansion" ist etwas Neues. Erstmals müssen keine Befehle mehr eingetippt werden; Aktionen werden durch die Kombination einer Reihe abgedruckter Verben mit den Elementen der Grafik ausgeführt: Wenn mit einem Schlüssel eine Tür geöffnet werden soll, klickt man auf das Wort "Benutze”, kramt aus den Hosentaschen (im Adventure-Jargon Inventar genannt) den Schlüssel hervor und klickt dann auf die Tür: „Benutze Schlüssel an Tür".

Die Einführung der CD-ROM bringt Neuerungen in zweierlei Hinsicht: Der große Speicherplatz und die weitflächige Etablierung von Soundkarten erlaubten, Spielen eine Stimme zu geben. Heute ist die Synchronisation aller Sprecher Standardmerkmal eines Adventures. Und dank der erhöhten Leistungsfähigkeit der PCs sorgten hochauflösende Standbilder oder gar gefilmte Animationen für Bewunderung. Als erstes Spiel, das speziell für CD-ROM entwickelt wird, wird 1993 "The 7th Guest" von Trilobyte bekannt. Doch das multimediale Zeitalter sorgt nicht nur für Freude. Ein Spielfilm ist zwar nett anzuschauen, eignet sich aber nicht sonderlich gut für Interaktionen. "The 7th Guest" ist im Grunde genommen nur eine Sammlung von Knobelaufgaben, die durch Videosequenzen miteinander verbunden werden. Die folgenden Spiele, bei denen Videos dominierten, zeigen endgültig, dass der Realismus mit verrin-

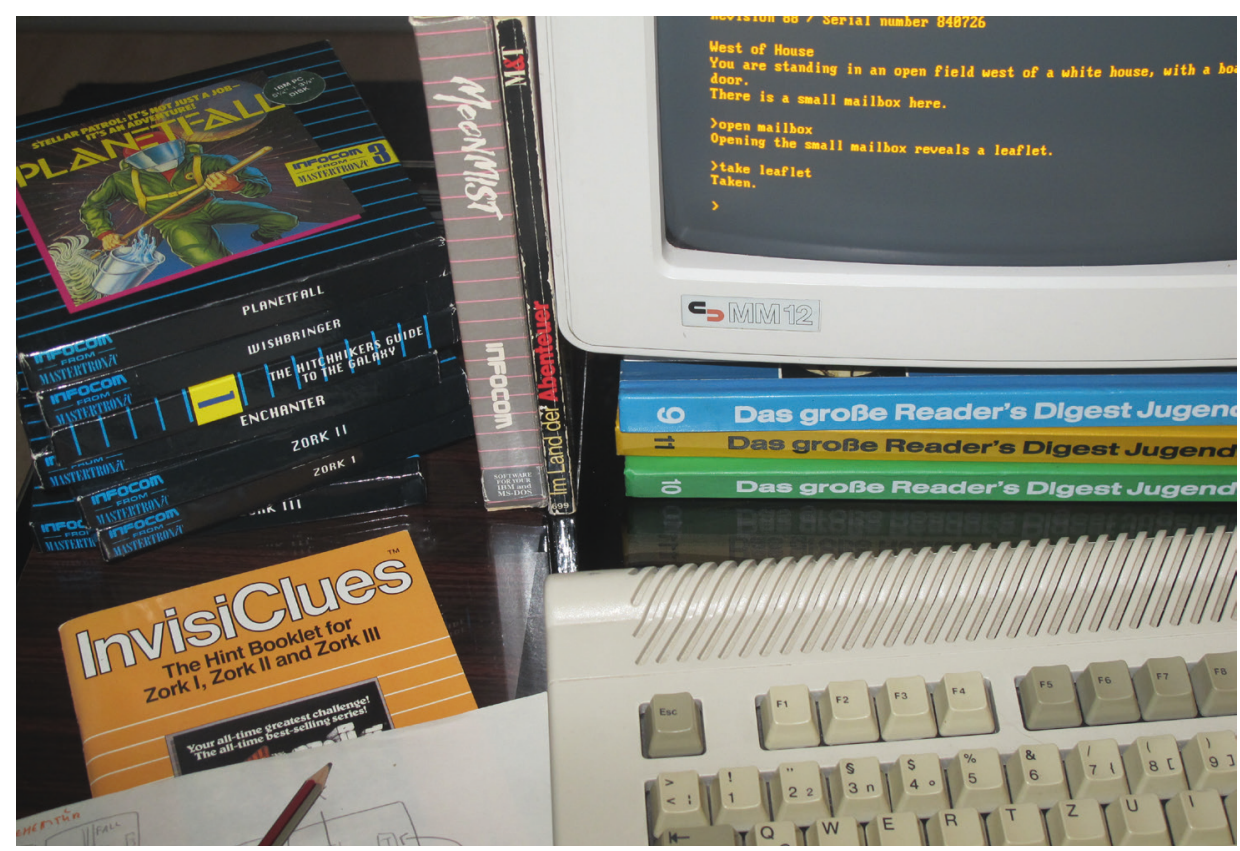

gerten Eingriffsmöglichkeiten erkauft wurde.

Heute besinnt sich die Industrie wieder auf gezeichnete Grafiken, wobei Auflösung und Farbtiefe verbessert wurden, wie etwa bei "Baphomets Fluch" von Revolution Software. In Deutschland ist für ihre guten Adventures wie "Edna bricht aus" vor allem Daedalic Entertainment bekannt.

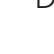




\section{Fußball}

Fußball ist bei Videospielen von Anfang an dabei: Bereits bei der ersten Spielkonsole, der Odyssey von Magnavox von 1972, kann um den Ball gekämpft werden. Zunächst nur Mensch gegen Mensch: Das Gerät kommt noch ohne elektronische Schaltkreise aus und ist nicht schlau genug, um einen Computergegner zu stellen. Auch um ein Spielfeld zu zeichnen, reicht die Kunst nicht. Daher werden durchsichtige Folien mitgeliefert, die man auf den Fernseher klebt. Der eigentliche Star der Odyssey ist aber ein anderes Ballspiel: Tennis. Es wird unter dem Namen "Pong" ein Verkaufsschlager - zunächst als Münzautomat von Atari, ab 1975 als Heimkonsole.

Neben TV-Konsolen sind seit den siebziger Jahren tragbare Geräte verbreitet. Die ersten Vorfahren des Game Boy haben einen winzigen Bildschirm und können nur einzelne Punkte oder Striche durch LEDs darstellen. Bei Football von Mattel (1977), einem der ersten Handhelds überhaupt, werden alle Spieler durch dünne rote Linien dargestellt. "Head to Head Soccer" von Coleco (1980) und "Soccer" von Tomy (1980) bieten bereits einen 2-Spieler-Modus.
Rasch entwickelt sich Fußball zu einem beliebten Spielgenre. Als erstes Spiel, für das der Name eines bekannten Fußballers lizenziert wird, gilt "Pelé's Soccer" von 1980 für das Atari VCS. Es ist das erste Spiel mit einem scrollenden Hintergrund.

Der Start der noch heute populären FIFA-Reihe ist "FIFA International Soccer" für das Sega Mega Drive 1993; bereits damals unter der Marke EA Sports. Mit „FIFA 16" werden Frauenmannschaften eingeführt; auf der US-Version des Covers ist mit Alex Morgan erstmals eine Frau zu sehen.
Die zweite große Marke unter den Fußballspielen ist "Pro Evolution Soccer" von Konami. Sie nimmt unter dem Namen "International Superstar Soccer" 1994 auf dem Super Nintendo ihren Anfang.

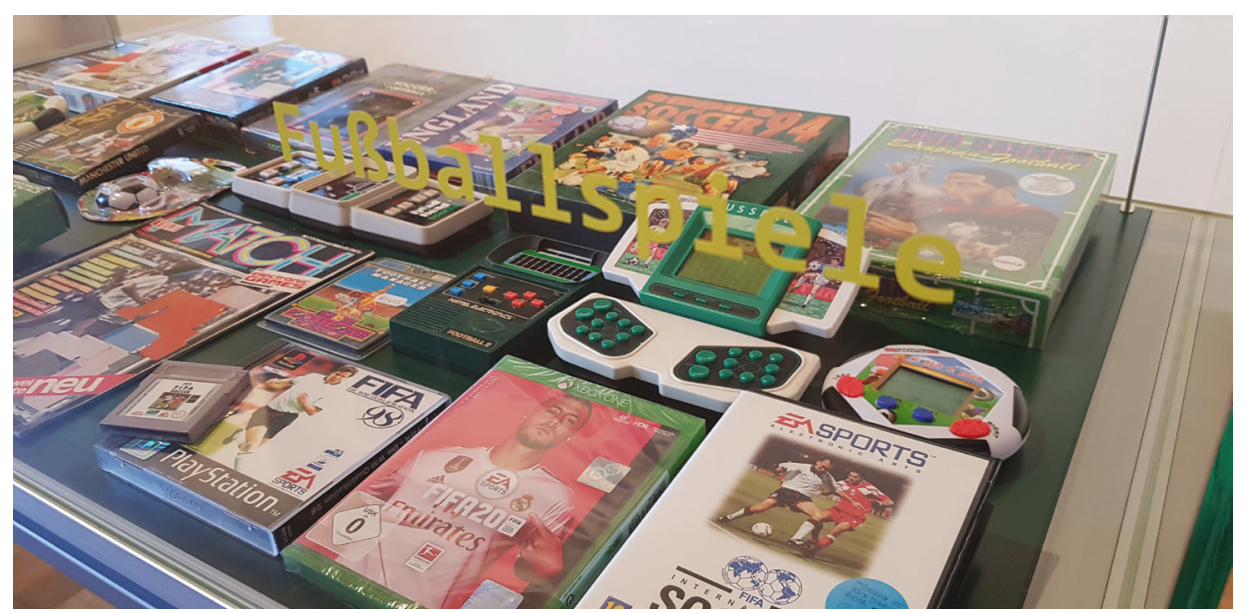




\section{Ralph reichts}

Spiele und andere Medien befruchten einander, was auch unter dem Fachbegriff Remediatisierung bezeichnet wird. Bücher und Filme werden als Spiele umgesetzt; aus Spielen werden Bücher und Filme, aus Filmen Serien oder Spiele. So thematisiert der Disney-Trickfilm "Ralph reichts" von $2012 \mathrm{Vi}$ deospiele und wird selbst als Videospiel für verschiedene Systeme umgesetzt. Synchronisiert wird der Titelheld durch einen Schauspieler: Christian Ulmen. Es gibt Bücher, ein Hörspiel und eine Soundtrack-CD. 2018 erscheint der Nachfolger "Chaos im Netz".

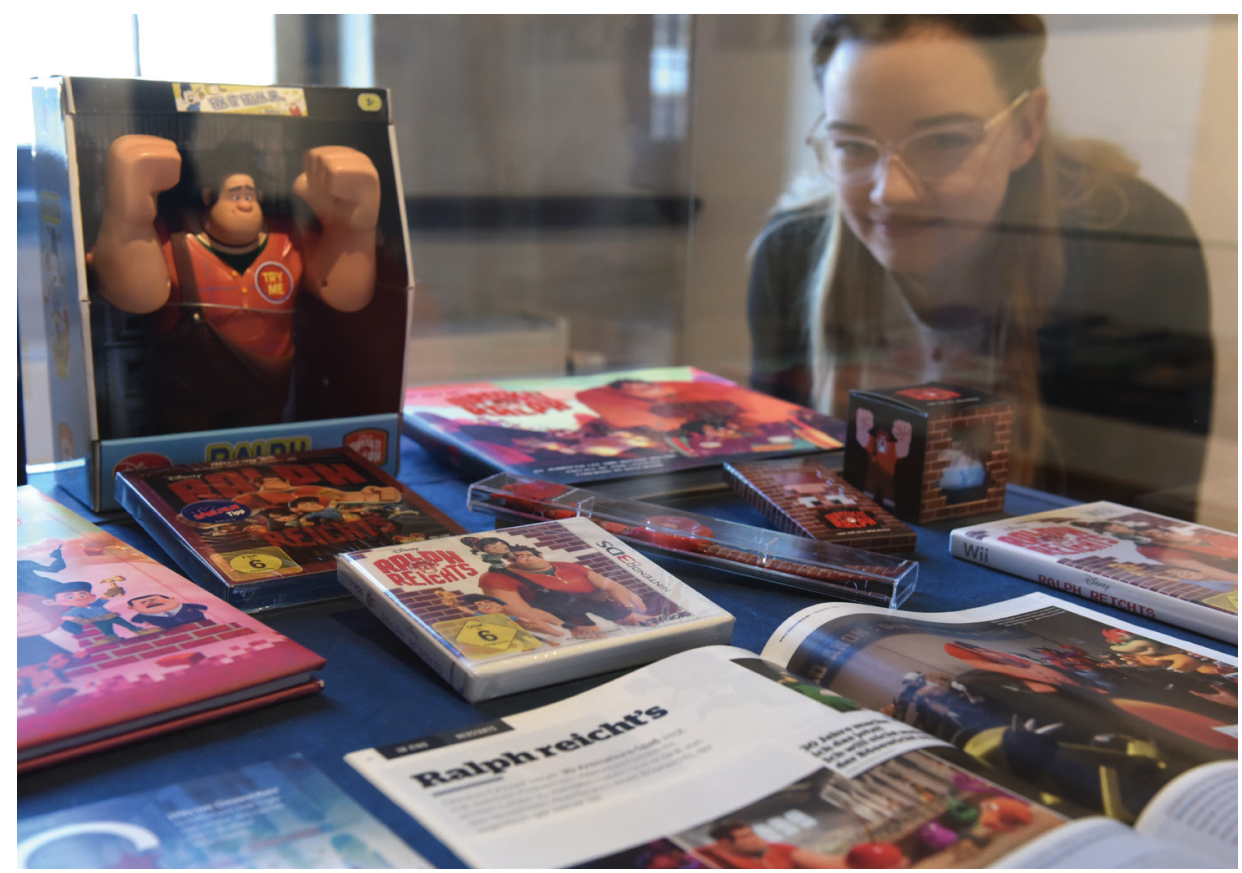




\section{Spielkonsolen der Ausstellung}

\section{Atari Video Computer System (1977) \\ BSS 01 \\ (1979)}

Die Urversion des Atari VCS 2600 erschien 1977 und begründete als erfolgreichste Konsole der 1970er und 1980er Jahre die Ära der Spielkonsolen mit austauschbaren Spielen (Spielmodule). Mit diesem Prinzip eröffnete Atari den Markt der Dritthersteller von Computerspielen, bis dahin produzierten ausschließlich die Hardware-Produzenten entsprechende Spiele, die auf den Konsolen und Automaten fest einprogrammiert waren und nicht getauscht werden konnten. Trotz einer vergleichsweise geringen Auflösung von 160x200 Pixeln wollten alle Spielinteressierten einen "Atari" haben, da durch das offene System auch ein großer Markt an Zubehör erhältlich war - angefangen von Spezial-Joysticks zur Steuerung bis hin zu Kopiersystemen für die Spielmodule. Das im Rahmen der Ausstellung mit dem Klassiker „Pac Man" spielbare "Junior-Modell" erschien gegen Ende der Produktionszeit für weniger als 50 US-Dollar.
Das Bildschirmspiel 01 (BSS 01) ist die einzige stationäre Spielkonsole der DDR, welche auf Grund ihres Preises (550 Mark der DDR) und inrer geringen Stückzahl (1000 Stück) nur selten in Privathaushalte gelangte. Die Konsole wurde vom Volkseigenen Betrieb Halbleiterwerk Frankfurt (Oder) zusammengebaut, Grundlage war der aus Westdeutschland importierte Mikrochip AY-3-8500 von General Instruments (USA): dieser wurde importiert, da das damals übliche Nachbauen von mikroelektronischen Bauteilen bei diesem Chip nicht realisiert werden konnte. Die Staatsführung der DDR wollte aber dennoch der Bevölkerung dem in westlichen Ländern populären Tennisspiel Pong! etwas Ebenbürtiges anbieten. In der BRD erhielt Pong! 1977 einen besonderen Schub durch die Abendsendung "Telespiele" mit Thomas Gottschalk, bei der Kandidaten am Telefon mit inrer Stimme den Schläger steuern. Auf dem BSS 01 waren vier Spiele auswählbar: Tennis (das originale Pong!), Fußball (jeder Spieler hat zwei Schläger, auf jeder Seite einen), Squash (beide Spieler spielen gegen die gleiche Wand), Pelota (Squash für einen Spieler). Ist keine der vier Spielarten-Tasten eingedrückt, erscheint ein verstecktes fünftes Spiel: Fußball mit Handicap. Hier hat der linke Spieler zwei Schläger, der rechte jedoch drei. Im Vergleich zum Original wurde das BSS 01 verbessert: über drei weitere Tasten kann die Ballgeschwindigkeit erhöht, der Ablenkwinkel verändert und die Schläger verkleinert werden.

\section{Kleincomputer KC85/3 (1984)}

Die Kleincomputer der Reihe KC 85 wurden ab 1984 in der DDR vom volkseigenen Betrieb VEB Mikroelektronik "Wilhelm Pieck” in Mühlhausen (Thüringen) aus dem Kombinat Mikroelektronik Erfurt gebaut. Die Kleincomputer sind in Schulen, Hochschulen und Arbeitsgemeinschaften, aber auch in Betrieben weit verbreitet, da sie erheblich preiswerter als Bürocomputer sind. Viele Anwender in der DDR sammeln mit innen ihre ersten Erfahrungen, da die meisten Geräte aber für Aufgaben der Volksbildung bestimmt und recht teuer sind (bis zu 4000 Mark der DDR), sind sie für private Anwender praktisch nicht verfügbar. Angetrieben wurde der Kleincomputer vom Prozessor U880 (mit 1,75 MHz-Taktfrequenz), welcher eine nicht lizenzierte Kopie des U.S.-amerikanischen Z80 (Zilog, San Jose, USA) von 1976 war. Der Z80 Kleinprozessor ist der bis heute am weitesten verbreitete 8-Bit-Prozessor weltweit und kommt u.a. auch in vielen Spielkonsolen wie dem Master System zum Einsatz. Verwirrenderweise gibt es Kleincomputer mit dem Namen KC 85 auch von Robotron Dresden. Das Werk in Mühlhausen bot eigene Software für ihren Kleincomputer an, teilweise auf Steckmodulen, vor allem aber auf Kassetten. Darunter auch Spiele-Sammlungen für je 38 Mark oder für einfache Programmierungen in der 1964 entwickelten Programmiersprache BASIC (= Beginner's All-purpose Symbolic Instruction Code"). 


\section{Master System (1987)}

1987 erscheint das Sega Master System und konkurriert damit auch außerhalb von Japan mit dem Nintendo NES. Die Konsole ist eine konsequente Weiterentwicklung des Sega SG-1000, von welchem mehr als 10 Millionen Stück verkauft wurden. Das Gerät wird von einem mit 3,58 MHz getakteten Prozessor angetrieben, der in 8 bit 16 Farben in einer Auflösung von 256x192 Pixel darstellen kann. Während in Japan verhältnismäßig wenig Spiele für das System erscheinen, ist dieses vor allem in Europa populär u.a. mit dem im Rahmen der Ausstellung spielbaren Jump-'n'-Run "Bubble Bobble”, bei welchem der Spieler seinen Gegner auf nebenund übereinander angeordneten Ebenen mit bunten Blasen beschießt.

\section{Nintendo Super Famicom (1989)}

Nintendos Super Famicom steht prototypisch für die rasante Entwicklung der westlichen Mikroelektronik vor allem der japanischen Hersteller von Spielkonsolen. Mit dem 16-fachen Arbeitsspeicher (128 kbyte) des Master Systems konnten nun 256 unterschiedliche Farben aus einer Auswahl von über 32.000 möglichen Farben dargestellt werden - in einer annähernd so großen Auflösung 512x448 Pixel wie die TV-Geräte der damaligen Zeit lag Nintendo damit auf der Höhe der Zeit Einen Schwerpunkt legte Nintendo vor allem auf den Sound, der in 16-bit-Stereo-Technik annähernd die Soundqualität von CDs lieferte. Das in der Ausstellung spielbare Spiel "Zelda" gilt als das erfolgreichste Action-Adventure um die beiden Helden "Link" sowie "Zelda", die Prinzessin des fiktiven Königreichs Hyrule. Die mittlerweile 19 Hauptspiele sind in unterschiedlichen Zeitepochen angesiedelt, auch die Spielemusikl verzaubert bis heute.

\section{Sony Playstation 1 (1994)}

Mitte der 1990er Jahre wurde Sony mit der "Playstation" der Weltmarktführer der Konsolenhersteller - mit mehr als 102 Millionen verkauften Konsolen. Die Konsole ist den Mitbewerbern (allen voran Sega) deutlich voraus und ermöglicht die Darstellung von 3D-Animationen in Echtzeit, sowie die fotorealistische Darstellung des Spielgeschehens mit mehr als 16 Millionen möglichen Farben. Als Speichermedium dient die Compact Disc - ReadOnlyMemory (kurz: CD-ROM), von welcher auch vorgefertigte Spielsequenzen und so genannte Cutscenes (Zwischensequenzen) als Videodatei abgespielt werden können. Die grafischen Qualitäten der Playstation 1 werden vom Publisher Capcom auch dazu genutzt, mit dem Spiel "Resident Evil" (USK 16/18) das Genre der Survival-Horror-Spiele einzuführen. In der Ausstellung ist mit dem Fußballspiel FIFA 98 (Electronic Arts) ein Vertreter der damals wie heute populären FIFA-Reihe zu spielen. Mit dem 1997 erschienene Spiel mit dem deutschen Fußballnationalspieler Andreas Möller auf dem Cover, konnte mit dem eingebauten Modem der Playstation 1 erstmals auch online mit anderen gespielt werden. 


\section{Kommunikation im Spiel}

Computerspiele sind nicht nur Unterhaltungsmedien, sondern im Wesentlichen Kommunikationsmedien, mit und über welche die am Spiel Beteiligten kommunizieren. Autoren und Programmierer vermitteln über Zeichen und Symbole im Spiel Informationen an die Spieler. Für das Zustandekommen dieser Kommunikation ist eine Rollenübernahme Voraussetzung. Das bedeutet, dass alle am Kommunikationsprozess Beteiligten über das gleiche Zeichen- und Symbolrepertoire verfügen müssen. Autoren, Programmierer und Produzenten von Spielinhalten müssen so auch die Kompetenzen und das Vorwissen der Spieler berücksichtigen und auf diese die Spiele und ihre Inhalte aufbauen - so werden bspw. üblicherweise die Spielfiguren in Computer- und Videospielen oft über die gleichen Tasten bewegt. Neuerungen, die keinen real-weltlichen Zeichen und Symbolen entsprechen, müssen bekannt gemacht werden. Dies geschieht meist in Tutorials zu Beginn des Spieles.

Kommunikation im Spiel findet auf mehreren Ebenen statt. Auf der Ebene des Spielinhalts können auch komplexe Inhalte sowie Wissen vermittelt werden bspw. über Geschichte sowie historische $\mathrm{Ab}$ läufe. Das Spiel "Anno 1701” (Sunflowers, 2006) im ersten Raum der Ausstellung ist ein prototypisches Beispiel für eine komplexe (Wirtschafts-)
Simulation, die in ihrer Gestaltung einer Welt in der frühen Neuzeit nachempfunden ist. Im Spiel kann sich der Spieler seine eigene Welt erschaffen. Notwendige Informationen über Steuereinnahmen, den Zustand der Bevölkerung oder die Produktivität einzelner Betriebe werden dem Spieler über eine Vielzahl von Icons angezeigt, auf welche der Spieler reagieren muss - in der Draufsicht auf die Spielwelt ("Gott-Perspektive") stehen inm dazu alle Möglichkeiten zur Verfügung, für welche es in unserer realen Welt auch Entsprechungen gibt (z.B. Handel mit anderen Völkern).

Auch innerhalb des eigentlichen Spieles spielt die Kommunikation eine wichtige Rolle. Herauszuheben ist "World of Warcraft" (Blizzard, 2004), oder einfach WoW genannt, in welchem die Spieler direkt mit einer Chat-Funktion miteinander kommunizieren müssen, um die Abenteuer der virtuellen Welt gemeinsam zu bestreiten. Das Prinzip der "Rollenübernahme" bestimmt alle Rollenspiele. Als Elfe, Heiler oder Wächter schlüpft der Spieler in WoW in eine Spielgestalt, die mit ganz unterschiedlichen Kräften und Kompetenzen ausgestattet ist. 2019 waren bspw. rund 11 Prozent aller Spieler Jäger, 10 Prozent Druiden und 9 Prozent Krieger. Spielziel und -ablauf ergeben sich in der offenen Spielwelt erst, wenn der Spie- ler mit anderen eine Gruppe bildet, um die virtuellen Abenteuer gemeinsam zu bestreiten. Ohne die direkte Kommunikation über die Chat-Funktion mit den weltweit zu Spitzenzeiten rund 9 Millionen Spielern kommt in WoW somit auch das eigentliche Spiel nicht zustande. Für das Spiel notwendige Ausstattungsgegenstände sowie Spielstände können innerhalb des Spieles auf diese Weise auch an andere Mitspieler übertragen oder sogar verkauft werden. In diesem Graubereich des Rechts geht es heute auch um Fragen, inwieweit virtuellen Informationen oder Daten (= Kommunikate) Eigenschaften von Gegenständen zugestanden werden.

Auch für die Erzeugung von Emotionen beim Spieler spielt die Kommunikation im Spiel eine wichtige Rolle, dies spiegelt sich auch in den Alterseinstufungen der USK wider. $\mathrm{Ne}$ ben Geräuschen und Spiel-Sounds werden vor allem durch die Gestaltung der Spielcharaktere Stimmungen und Emotionen angeregt. Dabei bedarf es nicht zwingend der neuesten Techniken zur Erzeugung bspw. der bedrohlichen Atmosphäre. Auch eher klobig und einfach gezeichnete Spielcharaktere in Onlinespielen, die vergleichsweise wenig Hardwareleistung benötigen, können durch inre Gestaltung bestimmte Stimmungen anregen. Das Spiel „Dungeons \& Dragons Online" (Eberron Unlimited,

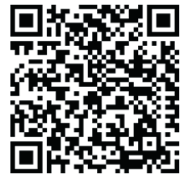


2006) zeigt beispielhaft, wie durch die Zeichnung von Drachen und Kämpfern, die eine mittelalterliche Anmutung haben, eine bedrohlich wirkende Stimmung erzeugt werden kann. Sie Details der Charaktere (bspw. Schuppen der Drachen, Kettenhemd des Kämpfers) regen die Phantasie der Spielenden an. Die aus der Filmgestaltung den Spielern bekannte Untersicht auf den gefährlichen Drachen soll zudem das Gefühl der Bedrohlichkeit verstärken.

Auch für die Spielmechanik spielt die Kommunikation mit dem Spieler eine entscheidende Rolle. Dies geschieht über direkte oder indirekte Hinweiszeichen im Spiel. Sogenannte "Cues" zeigen dem Spieler an, wohin er innerhalb der virtuellen Spielwelt seine Spielfigur bewegen soll. In dunklen und eher begrenzten Räumen und Gebäuden können dies neben Pfeilen auch ausgeleuchtete Wege sein. Gerade aber in den großen Spielwelten von Open-World-Games oder von Rollenspielen ist dies auch für die Orientierung in der Spielwelt wichtig. "The Witcher 3" (CD Project, 2015) zeigt prototypisch, wie durch den Lichteinfall der Sonne in der riesigen und äußerst realistisch dargestellten Märchenwelt für den Spieler oder dem Spielverlauf wichtige Details hervorgehoben werden.
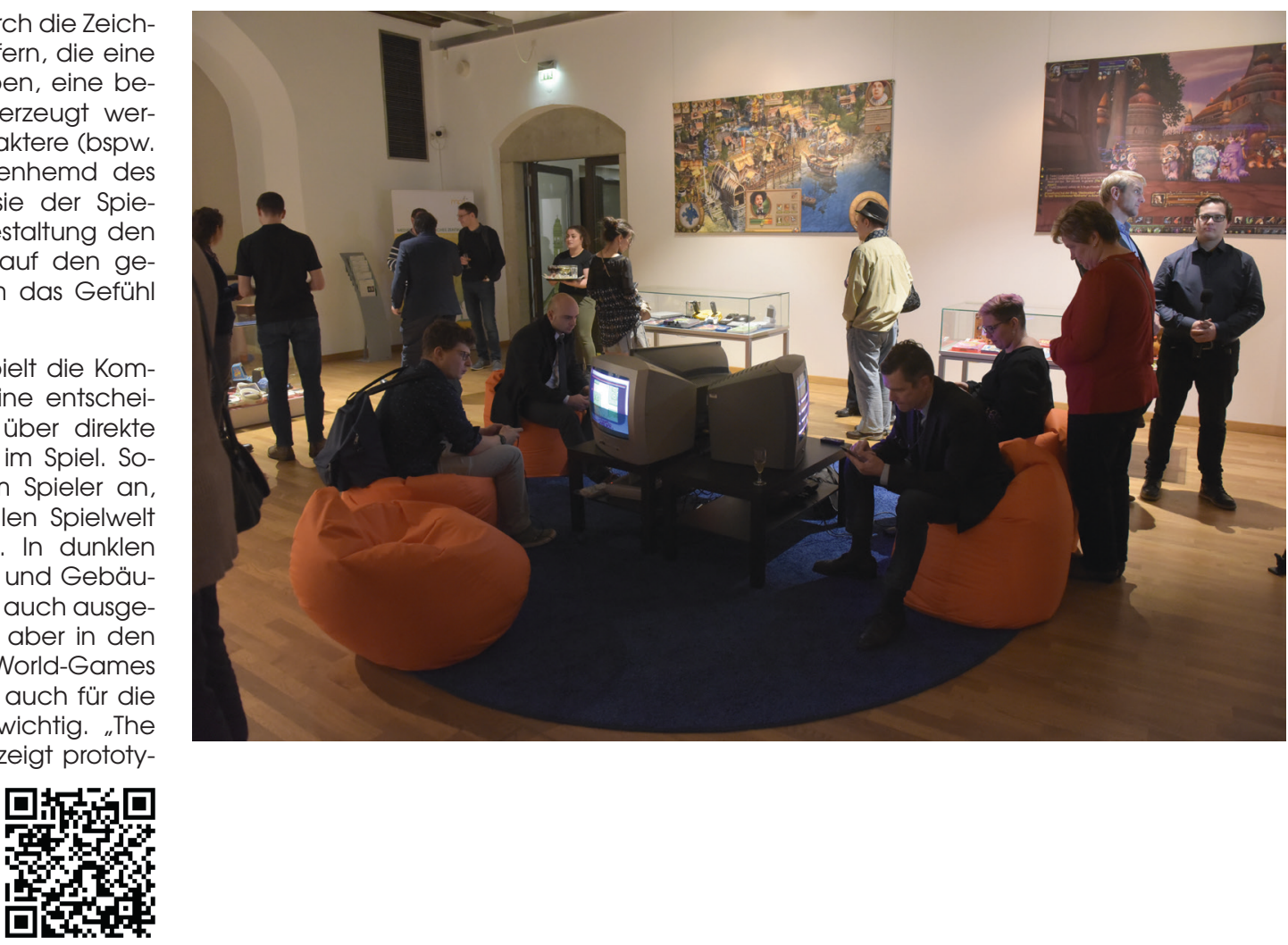


\section{Computerspiele als virtuelle Welten}

Antrieb der Entwicklung von Medien waren neben den technischen Errungenschaften und Entwicklungen schon immer auch die (künstlerischen) Bemühungen des Menschen, die Wirklichkeit detailliert abzubilden. Für die Kommunikationswissenschaft ist Wirklichkeit das, was in unserer Welt tatsächlich geschieht und vor allem das, was von den Medien beobachtet wird und worüber sie berichten

Sechs unterschiedliche Welten, in denen Menschen leben und integriert sind, können unterschieden werden: die reale Welt, die Traumwelt, die mentale Welt, die Spielwelt, die mediale Welt und die virtuelle Welt (Fritz 2004). Alle diese Welten sind Teil der Realität des Menschen, jede dieser Teilwelten funk tioniert nach eigenen Regeln und Charakteristika. Die Erfahrungen in den jeweiligen Teilwelten tragen dazu bei, dass sich die Wirklichkeit (Realität) durch neue Sichtweisen und Erfahrungen in diesen Teilwelten in unserem Bewusstsein weiter entfalten kann.

Die reale Welt stellt bspw. die gelebte Wirklichkeit des Einzelnen dar und lässt sich in die Bereiche der Umweltwahrnehmung sowie der Körperwahrnehmung einordnen. Die mediale Welt ist schließlich die Welt der (medialen) Informationsvermittlung (bsw. Presse), während die virtuelle Welt als Gegenpool jene Welt darstellt, in der bspw. Spieler von Computerspielen die "Allmacht” des konsequenzlosen Spielens erfahren und erleben können. Je mehr Sinne des Menschen angesprochen werden (bspw. 3D-Brillen), desto wirkmächtiger wird die Erfahrung der
Spielwelt und desto tiefer kann der Spieler in die Spielwelten von Computerspielen eintauchen. Die virtuelle Welt der Computerspiele kann all das ermöglichen, was weit über den Fähigkeiten und Möglichkeiten der Realität liegt, da sie nicht an die Regeln der realen Welt gebunden ist - der Imagination sind in dieser Welt letztlich keine Grenzen gesetzt. Virtuelle Welten sind in dieser Perspektive ein Produkt menschlicher Tätigkeit nämlich des Spielens

Die Spiele der Banner im zweiten Ausstellungsraum geben einen Überblick über die Möglichkeiten, wie die virtuellen Welten der Computerspiele mit den Möglichkeiten, die Wirklichkeit zu interpretieren und wiederzugeben, umgehen.

Die im Comic-Stil gestalteten Umgebungen und die humorvolle und gewaltfreie Umsetzung von "Monkey Island" (Lucasfilm Games, 1990) ist ein besonderes Beispiel, welches die Nähe zu den Fantasiewelten von Comics illustriert.

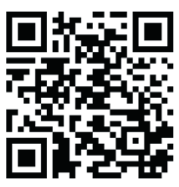

Die Nähe zu Märchen wird beim Rollenspiel „Drakensang" (Radon Labs, 2008) und der Zeichnung einer märchenhaften, mittelalterlichen Landschaft offensichtlich Rollenspiel(welten) wie diese sind eine konsequente Weiterenwicklung der mit Stift und Papier zu spielenden klassischen so genannten Pen-\&-Paper-Rollenspiele.
Das ebenfalls gezeichnete noch unvollendete "Star $\mathrm{Ci}$ tizen" (Cloud Imperium Games, N.N.) hingegen fokussiert auf eine in der fernen Zukunft spielenden Geschichte im Weltraum. Als MMO (Massively Multiplayer Online Game) greift es Elemente der klassischen Sciene-Fiction-Welten aus der Literatur (u.a. Stanislaw Lem) und dem Kino (u.a. Star Wars) auf.

Auch Ego-Shooter greifen häufig die Thematisierung einer fernen, utopischen und oftmals äußerst gewalttägen Zukunft auf. "Half Life 2" (Valve, 2004) erzählt vom Widerstand gegen ein totalitäres System, welches nach der Ankunft von Außerirdischen auf der Erde durch versehentlich geöffne-

口而制口 $14+2$ 5istin

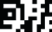

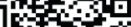
te Dimensionstore entstanden ist. Das Spiel ist damit auch ein herausragendes Beispiel, wie gesellschaftspolitisch kritische Entwicklungen auch in Spielen thematisiert werden können - trotz der expliziten Gewaltdarstellungen.

Diese Thematisierungen können auch in der Jetztzeit angesiedelt sein, wie das Beispiel "Far Cry 5" (Ubisoft, 2018) zeigt: im U.S.-amerikanischen Hinterland hat eine Sekte die Macht an sich gerissen und unterdrück† die Bevölkerung mit Waffengewalt und Drogen. Wie es typisch ist für diese Serie, greift auch die Spielfigur zur Waffe.

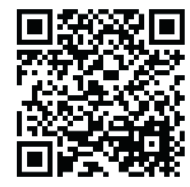


Computerspielwelten spielen häufig auch mit den Naturgesetzen: Die Überzeichnung der Kräfte der Physik zeigt besonders "Trials Rising" (RedLynx, 2019). Mit viel Fingerspitzengefühl muss der Spieler als Motoradfahrer durch haarsträubende Parcours fahren und springen - die Hindernisstrecken werden immer kniffliger. Selbst bei fehlerfreier Fahrt muss es am Ende nicht

gut ausgehen, was das Spiel detailliert und in Zeitlupe im Replay selbstironisch zeigt.

Die genannten Beispiele betonen zwei wichtige Aspekte von virtuellen Computerspielwelten:

Erstens bieten sie Möglichkeiten der gefahrlosen Exploration und des Ausprobierens. Jeder kann in die Rolle eines Helden schlüpfen, der je nach Spiel mit fantastischen Kräften ausgestattet sein kann. Computerspiele ermöglichen somit den Spielern auch, direkt und unmittelbar in die Handlung oder den Ablauf in die Abenteuer der Spielwelt eingreifen zu können. Gerade dieses direkte Feedback auf den Spieler - in der Wissenschaft "Selbstwirksamkeitserleben" genannt - ist eines der zentralen und motivierenden Aspekte, ein Spiel zu spielen.

Zweitens ist es unter medienpädagogischen Gesichtspunkten notwendig, darauf zu achten, daß vor allem Kinder und Jugendliche auf dem Weg der Aneignung des Spielens

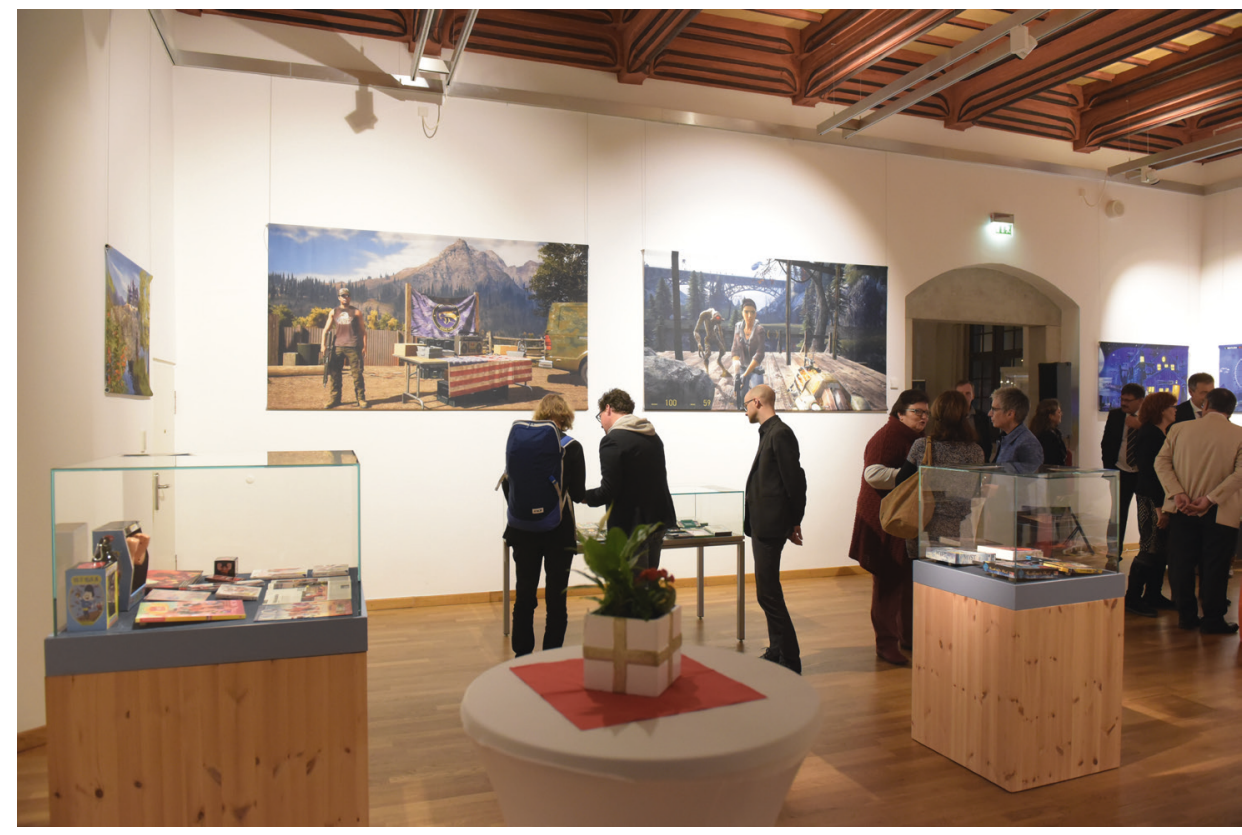

und der Inhalte dieser virtuellen Spielwelten begleitet werden, da sich die Themen, Inhalte und vor allem die Inszenierung von der realen Welt unterscheiden können.

der realen und virtuellen Welt hinreichend erkennen und die vermittelten Inhalte auch beurteilen können.

Die Alterskennzeichen der USK bieten hierbei eine wichtige Orientierung, ob das entsprechende Spiel für ein bestimmtes Alter geeignet ist. Nur so ist sichergestellt, daß Kinder und Jugendliche die Unterschiede zwischen 


\section{Faszination \& Bedeutung von Computerspielen}

7-teiliger Interviewfilm (38 Minuten) zur Ausstellung mit:

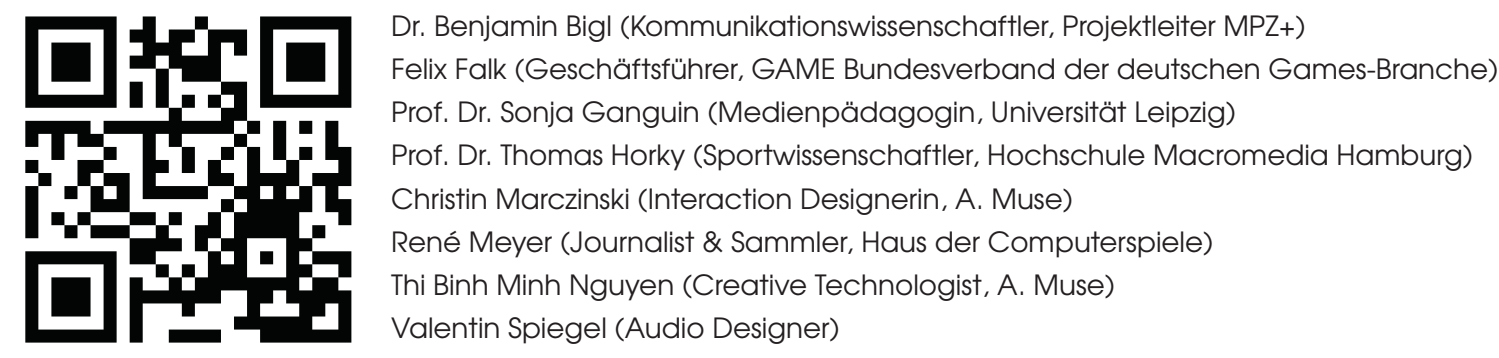

www.youtu.be/-xalmx19w0o

1. Anfang - Eindruck - Wirkung

2. Wirtschaft - Ausbildung - Potenziale

3. Kreation - Prozess - Ziele

4. Kultur - Geschichte - Gesellschaft

5. Medien - Verantwortung - Kompetenz

6. Sport-Anerkennung-Politik

7. Trends - Entwicklung - Zukunft

Redaktion: Benjamin Bigl \& Sascha Kummer

Kamera \& Schnitt: Sascha Kummer, www.la-maree.de 
Impressionen der Ausstellungseröffnung - 16. Dezember 2019

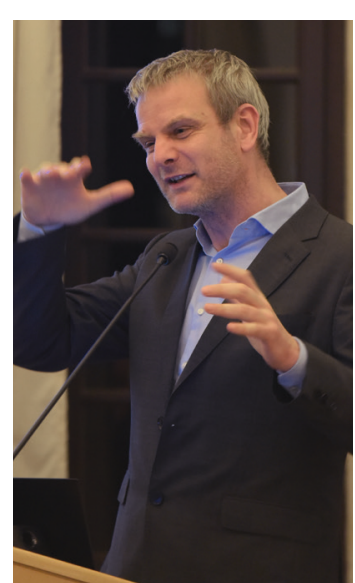

Prof. Dr. Markus Heinker (Präsident Sächsische Landesanstalt für Rundfunk und Neue Medien)
Dr. Benjamin Bigl

(Projektleiter MPZ+)

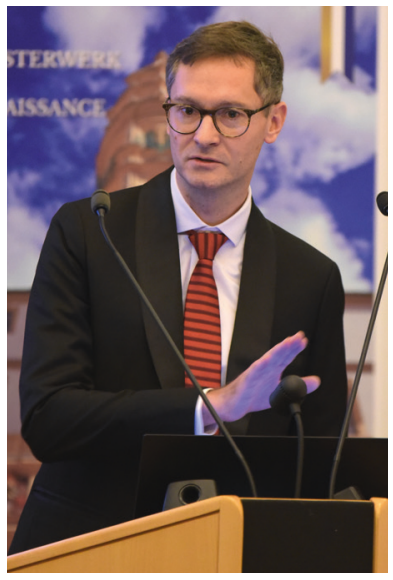

Prof. Dr. Jeffrey Wimmer

(Universität Augsburg)

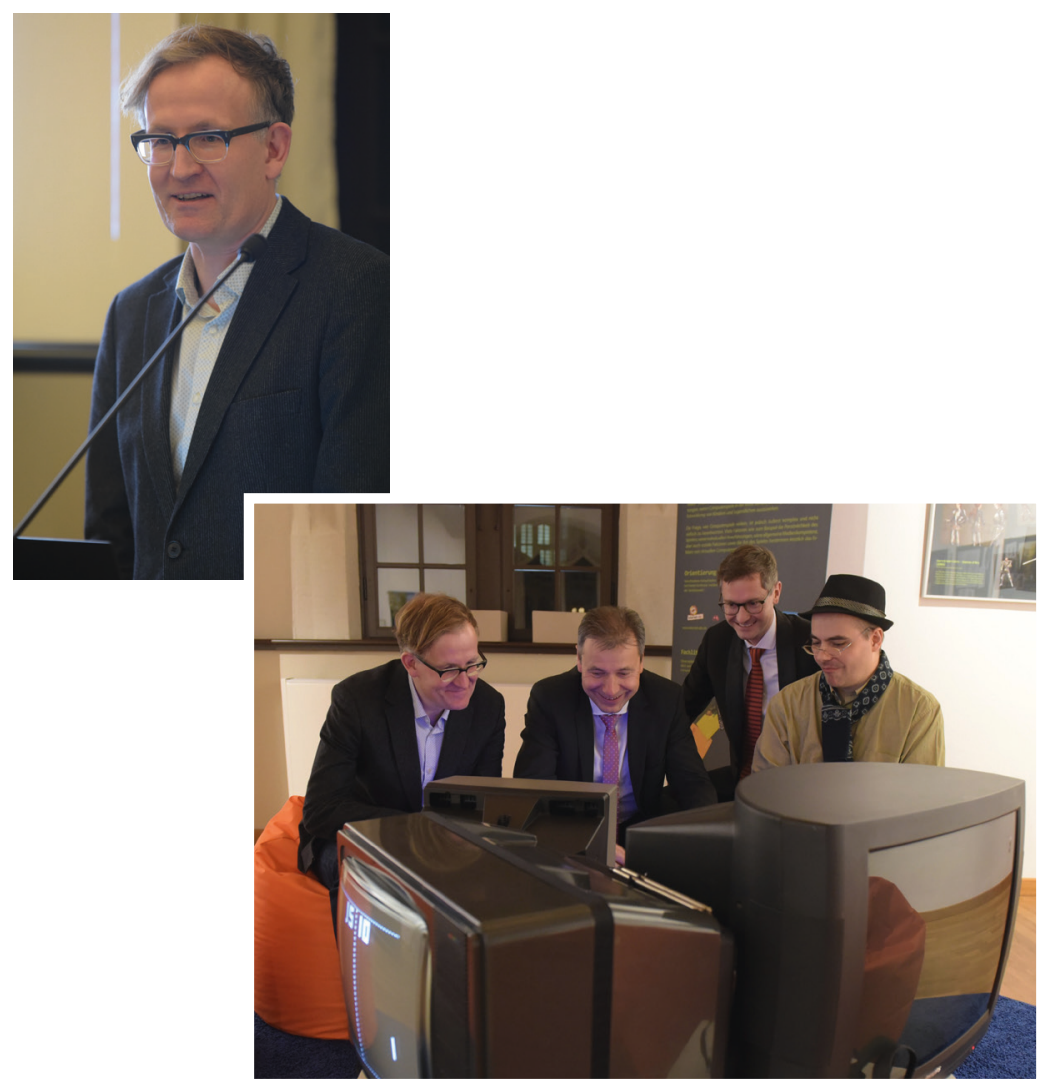

Prof. Dr. Jeffrey Wimmer (Universität Augsburg), Landrat Kai Emanuel, Dr. Benjamin Bigl (Projektleiter MPZ+), René Meyer (Haus der Computerspiele). 


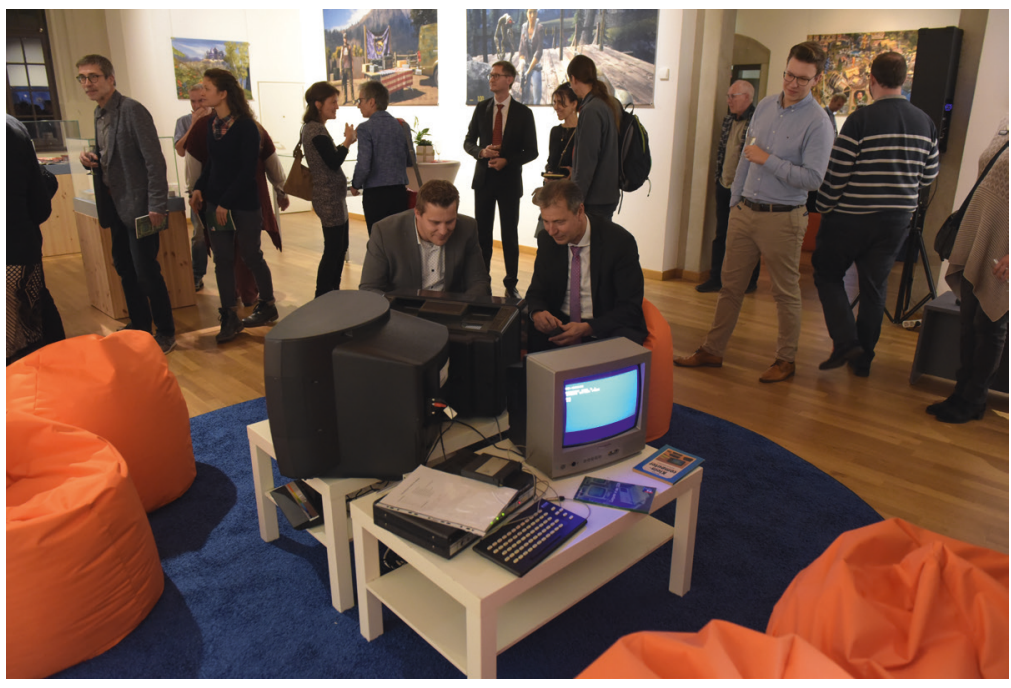

Film der Ausstellungseröffnung

La Maree Film, S. Kummer

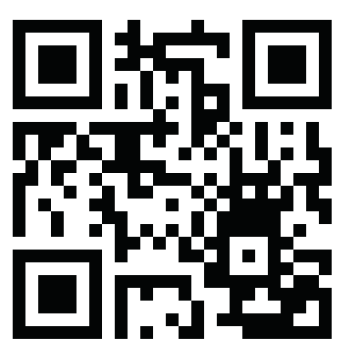

www.youtu.be/6uR1N-qMdOo
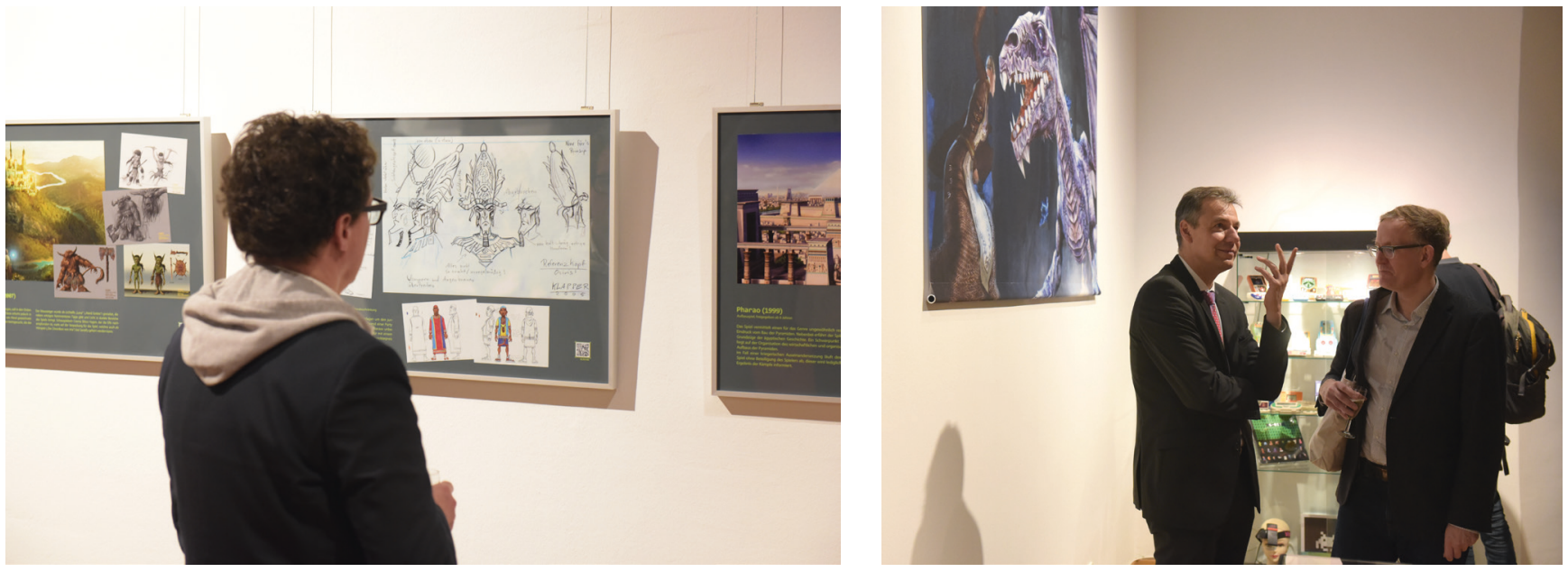


\section{Literatur}

Baacke, D. (1973). Kommunikation und Kompetenz. Grundlegung einer Didaktik der Kommunikation und ihrer Medien. München: kopaed.

Bareither, Christoph (2016): Gewalt im Computerspiel. Facetten eines Vergnügens. Bielefeld: transcript Verlag.

Beil, Benjamin; Hensel, Thomas; Rauscher, Andreas (Hrsg.) (2016): Game Studies. Berlin: Springer.

Bigl, Benjamin (2016): Virtuelle Computerspielwelten. Rezeption und Transfer in dynamisch-transaktionaler Perspektive. Köln: von Halem.

Fritz, Jürgen (2004): Das Spiel verstehen. Eine Einführung in Theorie und Bedeutung. Köln: Juventa.

Forster, Winnie (2009): Spielkonsolen und Heimcomputer. 1972-2009. Utting: Gameplan.

GAME - Verband der Deutschen Gamesbranche (2020). Marktdaten.

https://www.game.de/marktdaten/\#Zahlen\%20und\%20Fakten (2.1.2020)

Gierre, Daniel (2019): Computerspiele - Medienbildung - historisches Lernen. Zur Repräsentation von Geschichte in digitalen Spielen. Frankfurt am Main: Wochenschau Verlag.

Huizinga, Johan (2004): Homo Ludens. Vom Ursprung der Kultur im Spiel. Hamburg: Rowohlt.

Meyer, René (2019): Computer in der DDR. Erfurt: Landeszentrale für politische Bildung Thüringen.

Münte-Goussar, Stephan; Grünberger, Nina (2019): Medienbildung und die Kultur der Schule - Praxistheoretische Zugänge zur Erforschung von Schule in einer mediatisierten Gesellschaft. In Thomas Knaus (Hrsg.). Projekt - Theorie - Empirie. Spektrum medienpädagogischer Forschung. München: kopaed.

Sander, Uwe; von Gross, Friederike; Hugger, Kai-Uwe (2012): Handbuch Medienpädagogik. Berlin: Springer.

Statstia (2019): Verteilung der Spielercharaktere in World of Warcraft nach Klassen auf EUund US-Servern im Jahr 2019. https://de.statista.com/statistik/daten/studie/203876/umfrage/verteilung-der-world-of-warcraft-spielercharaktere-nach-klassen/ (2.1.2020)

Unterhaltungssoftware Selbstkontrolle (USK) (2020): USK-Alterskennzeichen. https://usk.de/alle-lexikonbegriffe/category/usk-alterskennzeichen/ (2.1.2020)

Verhovnik, Melanie (2015): School Schooting. Interdisziplinäre Analyse und empirische Untersuchung der journalistischen Berichterstattung. Baden-Baden: Nomos.

Wünsch, Carsten; Schramm, Holger; Gehrau, Volker; Bilandzic, Helena (Hrsg.) (2014): Handbuch Medienrezeption. Baden-Baden: Nomos. 
